University of Warwick institutional repository: http://go.warwick.ac.uk/wrap This paper is made available online in accordance with publisher policies. Please scroll down to view the document itself. Please refer to the repository record for this item and our policy information available from the repository home page for further information.

To see the final version of this paper please visit the publisher's website. Access to the published version may require a subscription.

Author(s): NATALIA L. KOMAROVA and SUZANNE J. M. H.

HULSCHER

Article Title: Linear instability mechanisms for sand wave formation

Year of publication: 2000

Link to published version:

http://dx.doi.org/10.1017/S0022112000008429

Publisher statement: None 


\title{
Linear instability mechanisms for sand wave formation
}

\author{
By NATALIA L. KOMAROVA ${ }^{1,2}$ \\ AND SUZANNE J. M. H. HULSCHER \\ ${ }^{1}$ Department of Mathematics, University of Warwick, Coventry, CV4 7AL, UK \\ ${ }^{2}$ Institute for Advanced Study, School of Mathematics, Einstein Dr., Princeton, NJ 08540, USA \\ ${ }^{3}$ Department of Civil Engineering, University of Twente, P.O. Box 217, 7500 AE Enschede, \\ The Netherlands
}

(Received 15 April 1998 and in revised form 13 January 2000)

A height- and flow-dependent model for turbulent viscosity is employed to explain the generation of sand waves in tidal seas. This new model resolves the problem of excitation of very long waves in sand wave formation, because it leads to damping of the long waves and gives a finite separation between the most excited mode and the zero mode. For parameters within their physically realistic ranges, a linear analysis of the resulting system yields a first excited mode whose wavelength is similar to the characteristic wavelength of sand waves observed in nature. The physical mechanism of sand wave formation as predicted by the new model is explained in detail. The dispersion relation obtained can be the starting point for a weakly nonlinear analysis of the system.

\section{Introduction}

The regularity of large-scale sea-bed patterns is rather striking. Ever since such patterns were first observed, it has been a challenge to explain their origin, spatial/ temporal scales and structure. There are many different kinds of periodic features which can be distinguished in tidal seas. At the largest scale we have tidal sand banks, huge elongated sand bodies spaced 5 to $10 \mathrm{~km}$ apart. Their orientation is slightly anticlockwise with respect to the prevailing tidal current. A recent review on the appearance and generation of sand banks is given by Dyer \& Huntley (1999). They can be described as free unstable modes appearing due to the interaction between horizontal tidal motion and the sandy sea bed (see Huthnance 1982; de Vriend 1990; Hulscher, de Swart \& de Vriend 1993; Hulscher 1996). Shore-face connected ridges (see van de Meene 1994) have roughly the same size as tidal sand banks, but they are oriented clockwise with respect to the tidal motion. Their generation can be explained by the interaction of the long-shore currents on a sloping bed, see Falques, Calvete $\&$ de Swart (1998). The subject of the present study is sand waves, see figure 1 . These are much smaller than sand banks and cover large areas of many sea beds, see e.g. Off (1963), Huntley et al. (1993), Katoh et al. (1998). These observations indicate that the spacing between sand waves varies between $100 \mathrm{~m}$ and $800 \mathrm{~m}$ and that their crests are more or less perpendicular to the main tidal current direction.

Tidal motion of water has been shown to be responsible for the formation of sand waves (Hulscher 1996). The main idea is as follows. If a periodic perturbation is applied to the bed, then the reaction of the time-periodic (tidal) flow will contain 


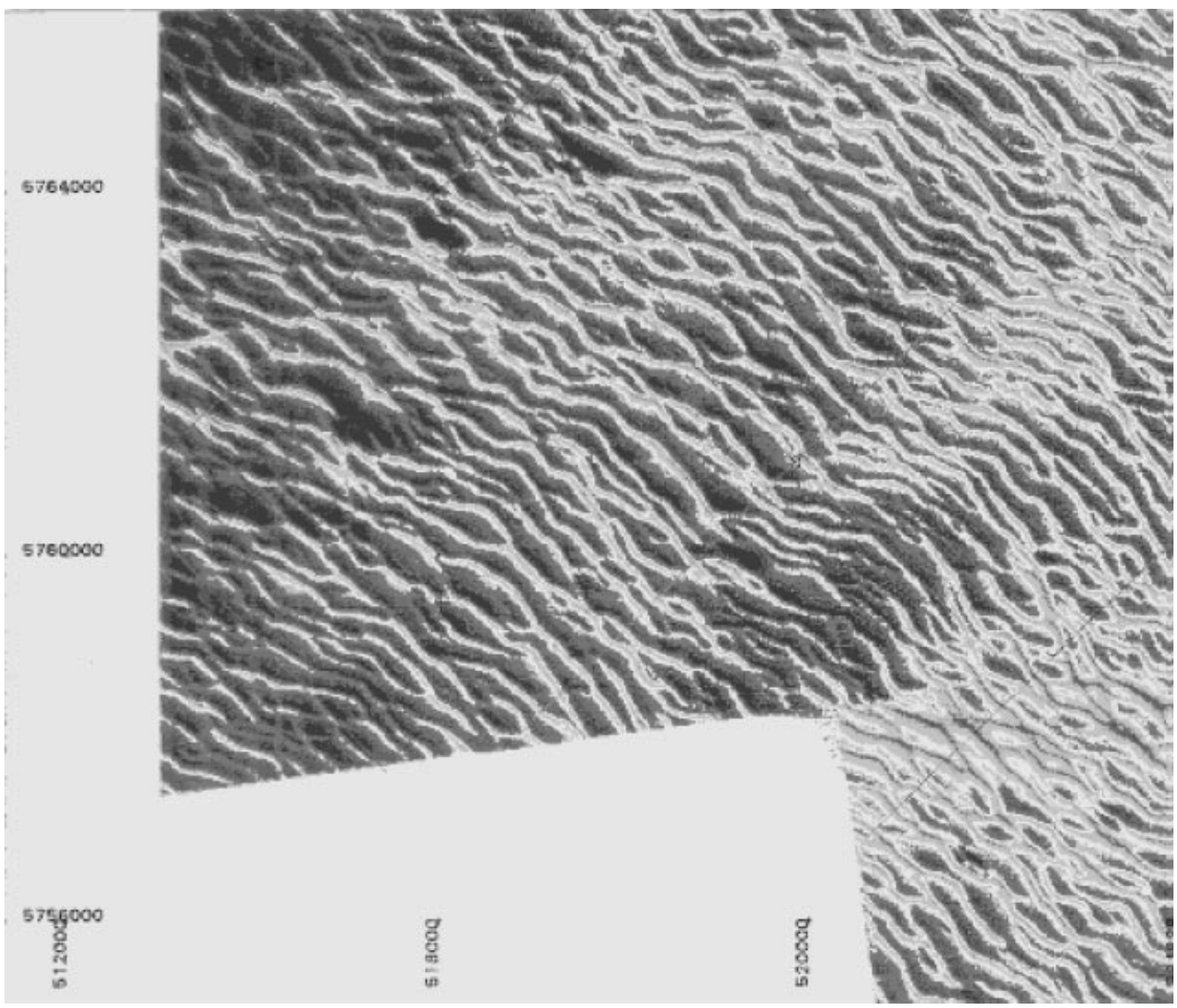

FiguRE 1. Sand waves near the Euro-channel (North Sea). After van Goor \& Andorka Gal (1996), courtesy of Rijkswaterstaat.

a time-independent component which is always directed towards the crests of the perturbation. This result has been reported in earlier works (for sand ripples, it was observed experimentally by Sleath (1976), and the exact solution for certain parameter ranges was given by Lyne 1971). In the context of sand pattern formation, the corresponding (truncated) solution was found by Blondeaux (1990) who studied small sand ripples created by sea waves, and by Hulscher (1996) who modelled the formation of sand waves in tidal seas. In all these cases, the time-averaged flow over the bed perturbation looks like circulating cells directed up the hill near the bed. The bed shear stress corresponding to these cells causes the growth of the perturbation. The counteracting mechanism is given by gravity, and the balance between the two defines the behaviour of the system. In the case of sand ripples, the system chooses a finite critical wavenumber which is excited if the currents are sufficiently strong. In the case of sand waves, no intrinsic wavelength was found in the linear analysis. This contradicts field observations which indicate the existence of a finite peak in the spectrum of sand waves.

In the model of Hulscher (1996) for tidal seas, the turbulent viscosity is assumed to be an external constant of the system. This leads to the excitation of a wide range of wavenumbers. As soon as currents are strong enough to overcome gravity, a bandwidth of wavenumbers between zero and some finite $k$ becomes excited, 


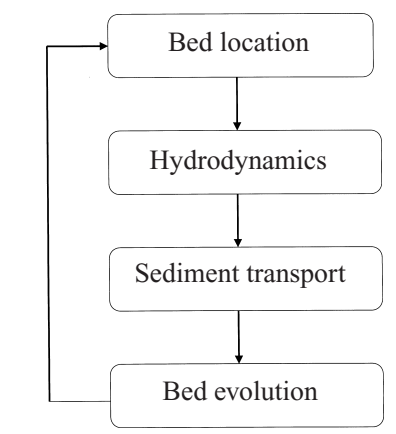

Figure 2. The morphological loop.

and ultra-long waves always have a finite growth rate. This non-physical behaviour suggests that the model of Hulscher (1996) is incomplete. Even though it contains the excitation mechanism responsible for the growth of sand waves, it fails to provide a mechanism for damping ultra-long waves.

In this paper, we describe a mechanism which leads to the choice of a finite wavelength in the pattern forming system. The main difference between our model and the model of Hulscher (1996) is that here we drop the assumption that the turbulent boundary layer is a constant. Bottom morphology interacts with the tidal flow and changes its properties. The viscosity parameterization proposed in this work reflects the fact that the turbulent boundary layer has a horizontal structure induced by deformations of the bed. We discuss a class of models where the eddy viscosity is a functional of the depth and the velocity, and give a set of conditions on the functional which provide a damping mechanism for ultra-long waves. It turns out that this mechanism is present in the flow as long as the eddy viscosity is larger in the troughs and smaller over the crests of the bed perturbation. We give a simple graphical explanation of the processes that take place in the system and explain why the depthdependent viscosity helps to suppress ultra-long waves. We also construct a simple example of a functional which possesses the necessary properties for suppressing the long waves and perform a linear analysis of the corresponding system. The resulting critical wavelength is similar to typical wavelengths of sand waves observed in nature.

In $\S 2$ we discuss the model. The basic tidal solution is given explicitly and a linear analysis is performed. Section 3 contains the results for a real physical situation. Section 4 is devoted to uncovering the physical mechanisms that lead to the bed instability. The role of the depth- and near-bed-dependent viscosity is explained from first principles. It is shown why the new viscosity mechanism helps to stabilize the very long waves. Section 5 presents some discussion and generalization of the results.

\section{Model}

In order to describe the formation of sand waves in a tidal sea, one needs to model the morphodynamic processes. This means that the equations of tidal motion for water and a model for sediment transport have to be specified, see figure 2. Once this is done, the coupling between the components of the morphodynamic loop is as follows. For a given bed level, the temporal and spatial variations of the sea water motion are described by the hydrodynamic model. These motions create shear stresses at the sea bed, which in turn make grains at the bed move. The bed shear stress is the relevant quantity for a bed-load sediment transport formulation. The spatial gradients 


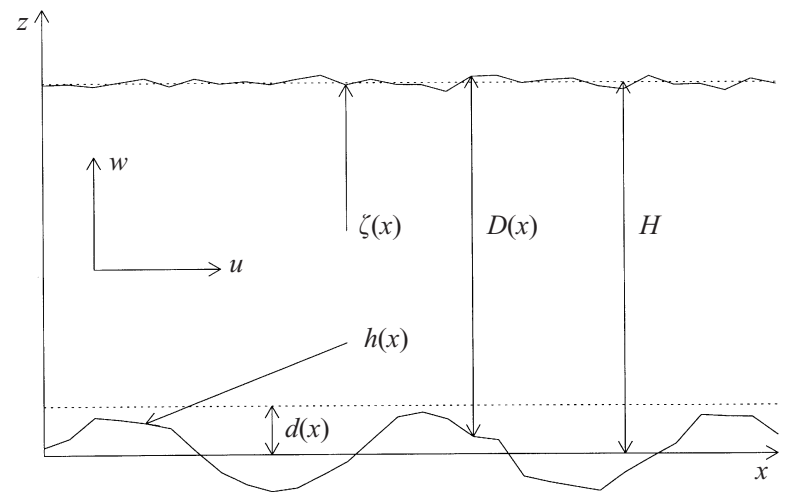

FIGURE 3. The geometry of the system and notation.

of the sediment transport cause local bed changes. Once the bed has changed, the new bed profile can be used as an input for the hydrodynamic equations, and the cycle can start again.

\subsection{Basic equations}

Sand wave crests are much longer than the spacing between them. In a threedimensional model it has been found that they are oriented almost perpendicular to the tidal motion and are only slightly affected by the Coriolis force (see Hulscher 1996). Therefore, we can restrict ourselves to one horizontal dimension and assume the bed to be homogeneous along the crests. Let $x$ be the horizontal axis (going across the wave crests) and $z$ the vertical one (pointing upwards). The bottom profile is located at $z=h(x)$ and surface at $z=H+\zeta(x)$ (note that $z=0$ and $z=H$ are the undisturbed bed and surface levels respectively, see figure 3). For the local (generally $x$-dependent) depth we introduce the notation $D$. Finally, $\boldsymbol{u}$ is the water velocity with components $(u, w)$ directed along $x$ and $z$. The hydrodynamic model which describes turbulent motion is given by the two-dimensional vertical Navier-Stokes equation,

$$
\begin{gathered}
\frac{\partial \boldsymbol{u}}{\partial t}+(\boldsymbol{u} \cdot \nabla) \boldsymbol{u}-\nabla \hat{\tau}=-\frac{\nabla p}{\rho}, \\
\nabla \cdot \boldsymbol{u}=0,
\end{gathered}
$$

where $\hat{\tau}$ is the shear stress matrix $\left(\begin{array}{cc}\tau^{x x} & \tau^{x z} \\ \tau^{z x} & \tau^{z z}\end{array}\right)$ with the Reynolds stress components

$$
\begin{aligned}
& \tau^{x x}=2 v_{t}^{h o r} \partial_{x} u, \quad \tau^{z z}=2 v_{t} \partial_{z} w, \\
& \tau^{x z}=\tau^{z x}=\left(v_{t} \partial_{z} u+v_{t}^{h o r} \partial_{x} w\right),
\end{aligned}
$$

(note that here and later in the text we will use three equivalent ways to denote partial derivatives: $f_{x} \equiv \partial_{x} f \equiv \partial f / \partial x$ and similarly with coordinate $z$ ). The stress components given above enter the momentum balance after averaging over the smaller-scale turbulent contributions in the advective terms (see Pedlosky 1987). In the equations above, $v_{t}$ and $v_{t}^{h o r}$ are the vertical and horizontal turbulent eddy viscosity components, $\nabla=\left(\partial_{x}, \partial_{z}\right), \nabla p$ is the horizontal pressure gradient and $\rho$ is the sea water density. It will turn out that the terms containing $v_{t}^{\text {hor }}$ do not play a significant role in the analysis, and only the vertical viscosity is important. In this paper we assume that the viscosity coefficient $v_{t}$ is independent of $z$. This assumption is commonly 
made despite being somewhat open to debate. In the next section we present two different models for the vertical viscosity. The boundary conditions on the sea surface, $z=H+\zeta(x)$, are given by

$$
\begin{gathered}
w=\frac{\partial \zeta}{\partial t}+u \frac{\partial \zeta}{\partial x} \\
\tau_{\|}=\tau_{\perp}=0,
\end{gathered}
$$

where $\left(\tau_{\|}, \tau_{\perp}\right)$ is the total shear stress. The boundary conditions at the sea bottom, $z=h(x)$, are

$$
\begin{gathered}
\tau_{\|}=S^{\prime} u, \\
w=\frac{\partial h}{\partial t}+u \frac{\partial h}{\partial x} .
\end{gathered}
$$

Equation (2.7) gives a relation between the bottom shear stress and the bottom velocity (see Maas \& van Haren 1987). It can be viewed as a 'relative' of the empirical expression $\tau_{b} \equiv u_{*}^{2}$, where $\tau_{b}$ is the bed shear stress and $u_{*}$ is the so-called friction velocity. However, equation (2.7) should not be confused with a linearization of this expression. In fact, the combination of equation (2.7) and a $z$-independent vertical viscosity is used as a model to describe the bottom shear stress and the velocity profile in a hydrodynamical system (see also Engelund 1970). In equation (2.7), $S^{\prime}$ is the resistance parameter $\left(S^{\prime}<\infty\right.$ corresponds to a non-zero slip, $S^{\prime}=$ resistance $=1 /$ slip). One could argue that $S^{\prime}=\infty$, the bed velocity $u=0$, is the most realistic condition. It is true in the case when the turbulent velocity, $v_{t}$, is taken to be a function of depth. However, in the present model, $v_{t}$ does not depend on $z$. The combination of $S^{\prime}=\infty$ and a $z$-independent eddy viscosity would give a greatly overestimated bottom shear stress, because $z$-independent viscosity models do not work well near the bed. Moreover, it is not the bed velocity which is responsible for the movement of the sand grains, but the bed shear stress, so the latter needs to be modelled in a correct way. Therefore, in physically realistic cases the resistance parameter $S^{\prime}$ must be finite.

Unlike depth-averaged models, the hydrodynamic model (2.1)-(2.8) explicitly gives the bed shear stress. This is the relevant quantity which determines the sediment transport, $q$. When modelling $q$, we choose to concentrate on the bed-load sediment transport and neglect the transport of suspended material. This is not a real shortcoming, as at offshore locations the bed-load transport is usually dominant. The bed-load sediment transport at the sea bed is modelled by

$$
q=\alpha^{\prime}\left|\tau_{b}\right|^{b}\left(\tau_{b}-\lambda^{\prime} \frac{\partial h}{\partial x}\right) .
$$

In this expression, $\tau_{b}$ denotes the volumetric bed shear stress (physical shear stress divided by the water density, hereafter we refer to it as bottom shear stress). Formula (2.9) is a generic expression that reflects the influence of the two most important forces which act on bed sediment grains. The first term shows the scraping effect of the drag force. The second term represents the gravity component along the bed profile; the effect is weighted by the down-slope coefficient $\lambda^{\prime}$. Finally, the proportionality coefficient $\alpha^{\prime}$ in combination with the nonlinearity parameter $b$ describes how efficiently the particles of sand are transported by the bed shear stress.

The particular expression for the sediment flux can be chosen in a number of ways (see, for instance, Fredsoe \& Deigaard 1992), but it turns out that as long as 
it contains both the 'drag force term' and the 'down-slope correction', the qualitative result will be the same. It is possible to get some intuition on the orders of magnitude using specific bed-load transport models which lead to expressions for the coefficients $b, \alpha^{\prime} \lambda^{\prime}$ as function of sediment characteristics (size, threshold angle, weight, etc). In Appendix B, some explicit estimates are given based on a Meyer-Peter-Muller model on a sloping bed in combination with threshold effects. The arguments given by Dyer (1986) motivate the choice $b=\frac{1}{2}$.

Following the morphological loop, we need to couple the hydrodynamic system with an equation describing sediment motion. This is given by a sediment mass conservation law, $\partial h / \partial t=-\partial q / \partial x$.

\subsection{Turbulence models}

Model (a). When studying large-scale morphological processes, the details of smallscale water motion can be effectively incorporated in the eddy viscosity coefficient, $v_{t}$ (see Pedlosky 1987). The simplest model for turbulent viscosity is $v_{t}=v_{0}$, a constant. It has already been shown by Hulscher (1996) that this gives qualitatively wrong behaviour of the dispersion curve for small wavenumbers $(k \rightarrow 0)$. Namely, the very long bed modes are the first ones to be exited. This means that the model fails to grasp some important physics in the system. Our suggestion is that this can be improved by taking into account the fact that viscosity depends on physical parameters of the system.

Model (b). We would like to motivate our choice of viscosity parameterization with the following intuitive considerations. The quantity $v_{t}$ defines the thickness of the turbulent boundary layer, $\sqrt{2 v_{t} / \sigma}$. For simple unidirectional or harmonic (tidal) flows over a flat bed, the assumption that this thickness is constant in time and space is not an unreasonable one. We will denote the (constant) eddy viscosity corresponding to an unperturbed tidal motion over a flat bed, as $v_{0}$. Now, for more complicated flows, the viscosity may become $x$ - and $t$-dependent. Let us assume that the unperturbed tidal flow is $u_{0}(z, t)=\tilde{u}(z) \sin (\sigma t+\Phi(z))$, and the bed distortion is periodic, e.g. $h(x)=2 \hat{h} \cos k x$. As water moves back and forth over bumps on the bed, the 'amount of turbulence' in water is likely to acquire a horizontal structure induced by the bed profile. The time-dependence of the flow will also affect the distribution of turbulent vortices. As long as the sand bumps are not too large, the viscosity changes induced by the bottom distortion are linear in $\hat{h}$. The following expression for the eddy viscosity summarizes these ideas:

$$
v_{t}=v_{0}\left(1+2 \hat{h}\left[\alpha_{1}(k) \cos k x+\alpha_{2}(k) \sin k x u_{0}(h, t)\right]\right) .
$$

The coefficients $\alpha_{1}$ and $\alpha_{2}$ in equation (2.10) could be made $z$-dependent but in this paper we will not consider this case. The $x$-dependence of the viscosity follows from the symmetries of the equations and the bed perturbation, i.e. $v_{t}$ should be invariant with respect to the change $x \rightarrow-x, u \rightarrow-u$. The first term in the square brackets in equation (2.10) represents time-independent changes in the eddy viscosity induced by the bed geometry. The maxima and minima of these changes must coincide with the extrema of the bed distortion. To prove this we note that the expression $\cos (k x-\chi)$ is invariant under the transformation $x \rightarrow-x$ only if $\chi=n \pi, n \in \boldsymbol{Z}$, i.e. the dependence must be $\pm \cos k x$, which is exactly in (out of) phase with the bed structure. The second term in the square brackets of equation (2.10) is the time-dependent part of the viscosity changes. It is induced by the time-periodic flow and therefore has the same time dependence as the tidal flow itself. Note that if $\hat{h}$ is small, then we 
can replace $u_{0}(h, t)$ by $u_{0}(0, t)$, because the difference is quadratic in $\hat{h}$. In a more general case, this term may have a phase shift with respect to the tidal flow (i.e. the time-dependence as $\left.\sin \left(\sigma t+\Phi^{\prime}\right), \Phi^{\prime} \neq \Phi(0)\right)$, or it could be a (possibly $z$-dependent) functional of the flow velocity. Again, equation (2.10) represents the simplest case. The spatial dependence of this term is periodic in $x$, but the maxima must be shifted with respect to the extrema of the bed profile by $\pi / 2$. This is again proven from the invariance under the transformation $x \rightarrow-x, u \rightarrow-u$.

The $k$-dependence of the coefficients $\alpha_{1,2}$ has to satisfy certain physical requirements. In model (2.10), $v_{t}=v_{0}=$ const for a flow over a flat bed $(\hat{h}=0)$. To be consistent with this, $\alpha_{2}$ must vanish at $k=0$, because situation $\hat{h} \neq 0, k=0$ corresponds to a flat bed as well. $\dagger$ On the other hand, the coefficient $\alpha_{1}$ tends to a finite constant as $k \rightarrow 0$, i.e. different (time-independent) values of viscosity correspond to different depths even in the case of a flat bed. The signs of $\alpha_{1,2}$ are easily interpreted. For instance, if $\alpha_{1}$ is negative, this means that the time-averaged viscosity is larger near troughs than near crests. If $\alpha_{2}<0$, it follows that the eddy viscosity is smaller on the downstream side of sand wave crests and larger on the upstream side (see figure 8). It is clear that the contribution from the second term in the square brackets of equation (2.10) is vanishingly small for ultra-long waves. Therefore, it is the term containing $\alpha_{1}$ which can make a difference for small values of $k$. It will turn out that if the coefficient $\alpha_{1}$ in expression (2.10) is negative for small values of $k$, this provides a mechanism for damping ultra-long sand waves.

An expression for eddy viscosity in terms of bulk parameters. As an example of parameterization (2.10), we propose a model where $v_{t}$ is expressed as a simple functional of the depth and the flow velocity. Our choice is based on the mixinglength concept, first introduced by Prandtl (1932). This means that the viscosity is thought of as proportional to the product of a typical velocity scale and a typical length scale (which gives the correct dimension). This reflects the fact that the amount of turbulence depends on the size of the vortices that can form in the sea, and on their velocity. The size of the vortices is restricted by the local depth, $D$, so the mixing length scale can be modelled by $D$. There are many ways of defining a characteristic value for the velocity which describes the rate of spinning of the turbulent vortices. As the turbulence in tidal motion is mainly bed-generated, the characteristic velocity should be measured near (or at) the bed. In the context of a $z$-dependent viscosity, the characteristic velocity scale is usually modelled by the friction velocity, $u_{*}$, see e.g. Tennekes \& Lumley (1972). In our case (a $z$-independent viscosity model), we use the near-bed velocity instead of the friction velocity in order to find the appropriate characteristic scale. We propose the following parameterization:

$$
v_{t}=c_{1} D\left|u_{n b}\right|_{\text {trunc }},
$$

where $c_{1}$ is a dimensionless constant, $D$ is the local depth and the near-bed velocity, $u_{n b}=u(z=d)$, is defined as the horizontal velocity estimated at some point $z=d$ close to the bottom (see figure 3). The truncation procedure in expression (2.11) is described below (see item iv). It will be shown later that the direct dependence

$\dagger$ One can argue that for $k=0$, the last term in (2.10) vanishes even for finite values of $\alpha_{2}$ because it involves $\sin k x$. However, this fact is irrelevant and is a consequence of our choice of the origin. For a general periodic bed perturbation, $2 \hat{h} \cos (k x+\phi)$, the term responsible for time-dependent changes of viscosity is $2 \hat{h} v_{0} \alpha_{2}(k) \sin (k x+\phi) u_{0}(h, t)$. This does not vanish automatically as $k \rightarrow 0$, and therefore we need the assumption $\lim _{k \rightarrow 0} \alpha_{2}=0$. Another way to show this is to use formula (2.10) directly. We need $\lim _{k \rightarrow 0}\left\|\alpha_{2}(k) \sin k x\right\|=0$, where $\|\cdot\|$ is a norm, e.g., the $l_{\infty}-$ norm, $\|f(x)\|=\sup _{x \in \mathbf{R}}(|f(x)|)$. This can be only achieved if $\lim _{k \rightarrow 0} \alpha_{2}(k)=0$. 
on the local depth does not play a significant role and it is the near-bed velocity dependence which is important. In a tidal environment, an analogous eddy viscosity parameterization was proposed by Soulsby (1990), and for rivers by Engelund (1970). More details and alternative (zero-equation) models for eddy viscosity can be found, for example, in Rodi (1980). Note that the concrete value of $d$ in equation (2.11) is not important for this analysis as long as $d$ is of the order of the sand wave magnitude. The reason for this is that terms proportional to $d$ do not enter the linear problem, they only appear at higher orders (see also the comment after equation (2.31)).

Now we list and discuss the main properties of the phenomenological model (2.11).

(i) $v_{t}>0$, which reflects the physical meaning of viscosity.

(ii) $v_{t}$ is linear in $D$ and $\boldsymbol{u}$. This assumption is consistent with, e.g., Prandtl (1932) and Tennekes \& Lumley (1972).

(iii) $v_{t}$ is $z$-independent. We introduced this limitation for simplicity. This assumption can be dropped for more refined models.

(iv) The time-dependence of $v_{t}$ cannot be more complex than the dynamics of the flow. Indeed, the quantity $v_{t}$ defines the vertical distance over which the flow changes. Therefore, its time-dependence has to be 'slaved' to the time-dependence of the flow. In the case of a periodic (tidal) flow containing only the first $n$ time-harmonics, this can be interpreted as follows: the expression for the turbulent viscosity can be time-dependent, but it cannot contain time-harmonics higher than $n$. For instance, the expression for $|u|$ will contain all the time-harmonics, but the harmonics higher than $n$ cannot be allowed in the expression for viscosity. This follows from simple physical common sense: the turbulent boundary layer of a flow cannot change faster than the flow itself. Therefore, we need to truncate the expression for $v_{t}$ so that it only contains $n$ harmonics.

(v) If we linearize $v_{t}$ for small bed perturbations, it can be written in the form (2.10), where $\alpha_{1}$ and $\alpha_{2}$ are both functions of $k$. It will be shown that $\lim _{k \rightarrow 0} \alpha_{1}=$ const $<0$ and $\alpha_{2} \sim k$ for small $k$.

Our viscosity model is consistent with the choice of finite values of the resistance parameter, $S^{\prime}<\infty$. In case of zero slip (an infinite resistance parameter), $v_{t}$ would be vanishingly small. In general, the resistance parameter $S^{\prime}$ in equation (2.7) is an $x$-and $t$-dependent quantity. In this work, we assume that it depends on the characteristic velocity, $u_{n b}$, in the same way as $v_{t}$ does, i.e. for model $(a), v_{t}=v_{0}, S^{\prime}=S_{0}^{\prime}$; for model $(b), v_{t}=c_{1}\left|u_{n b}\right|_{\text {trunc }} D, S^{\prime}=c_{2}\left|u_{n b}\right|_{\text {trunc }}$. Here, both $c_{1}$ and $c_{2}$ are dimensionless constants.

Remarks. - Time-dependence. We choose our model in such a way that (i) $v_{t}$ depends on $h$, and (ii) $v_{t}(h=0)=v_{0}$ (a constant, see Appendix A). The former assumption is new and crucial, whereas the latter one is based on earlier, generally accepted, work on tidal motion (Ekman dynamics; see e.g. Pedlosky 1987; Maas \& van Haren 1987; Prandtl 1932). We know that models with time-independent $v_{t}$ work well for tidal motion over a flat bottom. However, it is useful to note that, as far as our analysis is concerned, $v_{0}$ may or may not be time-dependent. As long as $v_{t}$ becomes slightly bigger in the troughs of a periodic perturbation, the ultra-long suppression mechanism will be present, no matter what the time-dependence of $v_{0}$ is.

- The main difference between models $(a)$ and $(b)$. Model $(b)$ assumes that eddy viscosity depends on local depth. The difference with the earlier (constant) model is that there, $v_{t}$ did not change when the bed was perturbed. The fact that viscosity depends on $h$ will lead to a new growth rate curve, which is a physically reasonable 


\begin{tabular}{|c|c|c|}
\hline Quantity & Scale & Comments \\
\hline$x, z, h$ & $\delta$ & $\begin{array}{l}\delta=\sqrt{2 v_{0} / \sigma}, \text { the unperturbed turbulent } \\
\text { boundary layer thickness }\end{array}$ \\
\hline$u, w$ & $U$ & $U$, the tidal velocity amplitude \\
\hline$t$ & $1 / \sigma$ & $\sigma$, the radian tidal frequency \\
\hline$\zeta$ & $U^{2} / g=\hat{f} H$ & $\hat{f}$, the Froude number \\
\hline$\tau_{b}, \tau_{\|}$ & {$[\tau]$} & {$[\tau]=v_{0} U / \delta$} \\
\hline$p$ & {$[p]$} & {$[p]=\rho \sigma^{2} \delta^{2}$} \\
\hline
\end{tabular}

TABLE 1 . Scaling used in the model. Here, quantity $=$ scale $\times$ dimensionless quantity.

consequence of such a dependence. Mathematically this will be shown by a linear analysis.

- Robustness. The calculations of $\S \S 2$ and 3 are performed with the specific functional form (2.11). It is however important to note that the physical mechanism of ultra-long wave suppression described in $\S 4$ only relies on property (v) and not on the concrete form of the functional.

\subsection{Scaling and basic tidal solution}

Our aim is to model sand waves generated by tide-topography interaction. This motivates the scaling given in table 1 . The constant $\delta$, the (unperturbed) turbulent boundary layer thickness, is a characteristic depth over which the water profile changes in the vertical direction. The Froude number, $\hat{f}$, gives the (squared) ratio between the water velocity and the speed of small-amplitude surface waves in shallow water. We can rewrite equations (2.1)-(2.2) in dimensionless variables,

$$
\begin{gathered}
2 u_{t}-1 / v_{0}\left[v_{t} u_{z z}+2 \partial_{x}\left(v_{t}^{h o r} u_{x}\right)+v_{t}^{h o r} w_{x z}\right]+R\left(u u_{x}+w u_{z}\right)=-p_{x} R, \\
2 w_{t}-1 / v_{0}\left[2 v_{t} w_{z z}+\partial_{x}\left(v_{t}^{h o r} w_{x}\right)+\partial_{x}\left(v_{t} u_{z}\right)\right]+R\left(u w_{x}+w w_{z}\right)=-p_{z} R, \\
u_{x}+w_{z}=0,
\end{gathered}
$$

(we introduced the notation $R=2 U /(\delta \sigma)$ and used the assumption that viscosity is $z$-independent). The characteristic viscosity, $v_{0}$, is defined as the turbulent viscosity corresponding to the basic flow solution (see Appendix A). It is convenient to introduce the stream function, $\Psi$, so that $u=\Psi_{z}, w=-\Psi_{x}$. Then the above equations can be combined by eliminating the pressure gradients, and the system can be rewritten as

$$
R^{-1}\left[2 \partial_{t}\left(\partial_{z}^{2}+\partial_{x}^{2}\right)-v_{0}^{-1} \hat{\mathrm{N}}\right] \Psi=-\Psi_{z}\left(\partial_{z}^{2}+\partial_{x}^{2}\right) \Psi_{x}+\Psi_{x}\left(\partial_{z}^{2}+\partial_{x}^{2}\right) \Psi_{z},
$$

where $\hat{\mathrm{N}}$ is a linear operator depending on the viscosity,

$$
\hat{\mathrm{N}}=v_{t} \partial_{z}^{4}+2 \partial_{x} v_{t}^{\text {hor }} \partial_{x} \partial_{z}^{2}-v_{t}^{\text {hor }} \partial_{x}^{2} \partial_{z}^{2}+2 \partial_{x} v_{t} \partial_{x} \partial_{z}^{2}+\partial_{x}^{2} v_{t}^{\text {hor }} \partial_{x}^{2}-\partial_{x}^{2} v_{t} \partial_{z}^{2}
$$

The boundary conditions in dimensionless variables are

$$
\begin{gathered}
w=\hat{f}\left(\frac{\sigma H}{U} \frac{\partial \zeta}{\partial t}+\frac{H}{\delta} u \frac{\partial \zeta}{\partial x}\right), \quad \tau_{\|}=\tau_{\perp}=0 \quad \text { at } z=\frac{H}{\delta}(1+\hat{f} \zeta), \\
\tau_{\|}=\frac{S^{\prime} \delta}{v_{0}} u, \quad w=\frac{\partial h}{\partial t}+u \frac{\partial h}{\partial x} \quad \text { at } z=h .
\end{gathered}
$$


Note that in situations of interest, $H / \delta$ is of order one and $\sigma H / U$ is less than one. Finally, the sediment evolution equation in dimensionless variables is

$$
\frac{\partial h}{\partial t}=-\alpha \frac{\partial}{\partial x}\left(\left|\tau_{b}\right|^{b}\left(\tau_{b}-\lambda \frac{\partial h}{\partial x}\right)\right),
$$

where $\alpha=\alpha^{\prime}[\tau]^{3 / 2} /\left(\sigma \delta^{2}\right)$ and $\lambda=\lambda^{\prime} /[\tau]$, see Appendix B. We will make the following important approximations:

(i) Shallow water approximation: The geometry of our system is such that the relevant horizontal distances (the size of sand waves, which is of the order of hundreds of metres) are much larger than the vertical distances (the local depth is less than fifty metres). Since both the horizontal and vertical distances are scaled in the same way, in dimensionless variables this is manifested in the relation $\left\|\partial_{x}\right\| \ll\left\|\partial_{z}\right\|$. This allows us to neglect the second $x$-derivatives in equations $(2.12)-(2.13)$ in comparison with second $z$-derivatives. For tidal environments, it is very difficult to give reliable estimates for the horizontal viscosity coefficient. According to Okubo \& Ozmidov (1970), Ozmidov (1968), the value is of the same order of magnitude or slightly larger than the vertical viscosity. Indeed, in the horizontal direction one needs to resolve up to distances of about $100 \mathrm{~m}$ or less, which gives the estimate $v_{t}^{\text {hor }} \sim 10^{-1} \mathrm{~m}^{2} \mathrm{~s}^{-1}$ or less, and the vertical viscosity coefficient has the order of magnitude of $10^{-2} \mathrm{~m}^{2} \mathrm{~s}^{-1}$. Therefore, the effects of the horizontal viscosity in this case are very small. However, some authors give much larger estimates (see Beerens 1995, who uses $v_{t}^{h o r} \sim 1^{-2} \mathrm{~m}^{2} \mathrm{~s}^{-1}$ ). In this case, the horizontal viscosity plays a role. Fortunately, even if the latter estimate were the case, inclusion of this term would not add new behaviour in this model. Namely, the effect of the term with horizontal viscosity coefficient would lead to the damping of small bed forms for hydrodynamical reasons, and this behaviour is already included through the down-hill sediment transport mechanism. In order to keep the model as transparent as possible, we neglect the term with $v^{\text {hor }}$ from now on. Finally, in the shallow water approximation we can neglect the contribution $\partial_{x} u$ in comparison with $\partial_{z} u$ in the expression for the bottom shear stress, $\tau_{\|}$. Therefore, according to table 1 , in dimensionless variables, the shear stress is given by $v_{t} / v_{0} \partial_{z} u$.

(ii) 'Rigid lid' approximation: $\hat{f} \ll 1$. The surface boundary conditions are now evaluated at the constant height, $z=H / \delta$. Therefore, the parallel surface shear stress, $\tau_{\|}$, is proportional to $u_{z}$. This approximation also suggests that the surface boundary condition, $\tau_{\perp}=0$, simply means that the pressure is hydrostatic. Since the pressure does not enter equation (2.12), this condition does not enter the further analysis.

(iii) Quasi-stationary approach: $\Delta t_{\text {hydrodynamic }} / \Delta t_{\text {morphodynamic }} \ll 1$. This follows from the fact that $\alpha$ in equation (2.16) is a small quantity (see Appendix B). This physically means that the ratio between the tidal period and the morphological time scale is small. Therefore, one can neglect the time-derivative in the second equation (2.15). Also, one can treat the shape of the bottom as a fixed function of $x$ when solving the hydrodynamic problem, and then average the sediment balance over a tidal period in order to evaluate the long-term bed evolution (equation (2.16)). The relevant measure for the morphological time is

$$
\Delta T \equiv 1 /(\alpha \sigma) .
$$

In other words, if we denote the physical time (measured in seconds) as $t_{d i m}$, then the dimensionless hydrodynamical time variable and the morphological time variable are given by $t=t_{d i m} \sigma$ and $T_{m}=t_{d i m} / \Delta T$ respectively.

Finally, we can write down the full dimensionless model describing the dynamics of a water-sand system assuming the shallow water, rigid lid and quasi-stationary 

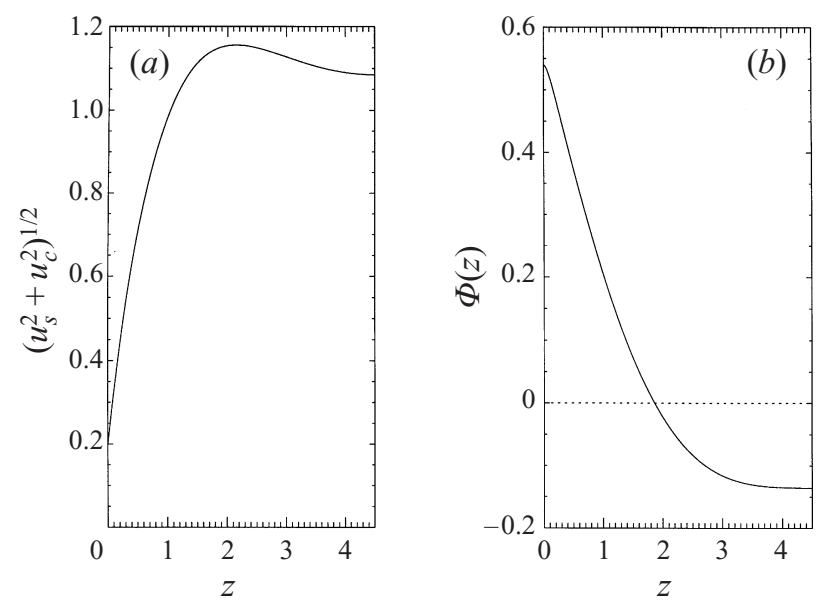

Figure 4. The components of the horizontal basic flow for $H=45 \mathrm{~m}, v_{0}=7 \times 10^{-3} \mathrm{~m}^{2} \mathrm{~s}^{-1}$, $S_{0}^{\prime}=4.7 \times 10^{-3} \mathrm{~m} \mathrm{~s}^{-1}$; in $(a)$ the amplitude, $\tilde{u}$, of the velocity is given, and in $(b)$ the phase shift in radians is plotted versus the vertical distance from the bottom.

approximations:

$$
\begin{gathered}
1 / R\left(2 \partial_{t}-v_{t} / v_{0} \partial_{z}^{2}\right) \Psi_{z z}=-\Psi_{z} \Psi_{x z z}+\Psi_{x} \Psi_{z z z}, \\
z=H / \delta: \quad \Psi_{z z}=0, \quad \Psi_{x}=0, \\
z=h: \quad \Psi_{z z}-S^{\prime} \delta / v_{t} \Psi_{z}=0, \quad \Psi_{x}=\Psi_{z} h_{x}, \\
\frac{\partial h}{\partial T_{m}}=-\frac{\partial}{\partial x}\left\langle\left|\tau_{b}\right|^{b}\left(\tau_{b}-\lambda h_{x}\right)\right\rangle,
\end{gathered}
$$

(the angular brackets denote the averaging over a tidal period).

System (2.18)-(2.21) has a solution which corresponds to an $x$-independent horizontal tide over a flat bed (i.e. the solution $\Psi$ does not depend on $x$, and $h=0$ ). In this paper we prescribe the time-dependence of the basic solution as an $M_{2}$-tide. In the subsequent analysis, all the higher harmonics (tides $M_{4}, M_{6}$ etc.) are neglected (see § 2.4). The problem for the tidal flow is solved explicitly in Appendix A. The solution can be written as

$$
u_{0}(z, t)=u_{s}(z) \sin t+u_{c}(z) \cos t \equiv \tilde{u}(z) \sin (t+\Phi(z))
$$

where

$$
\tilde{u}(z)=\sqrt{u_{s}^{2}(z)+u_{c}^{2}(z)}, \quad \tan \Phi(z)=u_{c}(z) / u_{s}(z) .
$$

These two quantities are shown in figure 4 as functions of $z$. One can see from figure $4(a)$ that the water velocity amplitude increases away from the bed (the characteristic distance over which it changes is defined by $\delta$ ). Note that at the bed it is not equal to zero because of the presence of a resistance parameter, $S^{\prime}<\infty$. Figure $4(b)$ gives the (relative) difference in time at which the maximum of the flow velocity is achieved. For a typical tidal period of 12 hours 25 minutes, the surface flow lags approximately $(0.7 / 2 \pi) \times 12 \mathrm{~h} 25 \mathrm{~min}=1$ hour 23 minutes behind the bed flow.

\subsection{Linear stability analysis}

In order to study the formation of rhythmic patterns in this model, we perturb the sea bottom so that the new bed profile looks like $z=\hat{h} \mathrm{e}^{\mathrm{i}\left(\omega T_{m}+k x\right)}+$ c.c., $\hat{h} \ll 1$. The 
local depth now varies spatially and is equal to $D\left(x, T_{m}\right)=H-\hat{h^{i}\left(\omega T_{m}+k x\right)}+$ c.c. The wavenumber $k$ characterizes the spacing of the crests, $-\operatorname{Im} \omega$ is the growth rate and $\hat{h}$ denotes the amplitude of the bed pattern. As the bed is perturbed, the flow pattern and (for model $b$ ) the turbulent viscosity change as well. Let us denote the relative change of the viscosity with respect to $v_{0}$ as $v \equiv v_{t} / v_{0}-1$. Then the perturbed quantities can be written as a vector,

$$
\left(\begin{array}{l}
\Psi \\
h \\
v
\end{array}\right)=\left(\begin{array}{c}
\Psi_{\text {basic }}(z, t) \\
0 \\
0
\end{array}\right)+\hat{h} \mathrm{e}^{\mathrm{i}\left(\omega T_{m}+k x\right)}\left(\begin{array}{c}
\Psi_{1}(z, t) \\
1 \\
v_{1}
\end{array}\right)+\text { c.c. }
$$

Note that the flow velocity perturbation, $\Psi_{1}$, is an unknown function, whereas the viscosity change, $v_{1}$, is a function of $\Psi_{1}$ (it is specified further in this section). The stream function, $\Psi_{1}$, satisfies the following Orr-Sommerfeld-like equation:

$$
(\mathrm{i} k R)^{-1}\left(\left(2 \partial_{t}-\partial_{z}^{2}\right) \Psi_{1 z z}-v_{1} u_{0 z z z}\right)=-u_{0} \Psi_{1 z z}+u_{0 z z} \Psi_{1},
$$

with the boundary conditions

$$
\begin{gathered}
z=H / \delta: \quad \Psi_{1 z z}=0, \quad \Psi_{1}=0, \\
z=0: \quad \Psi_{1 z z}-S_{0} \Psi_{1 z}=-\left(u_{0 z z}-S_{0} u_{0 z}\right)+\Gamma \delta / H u_{0}, \quad \Psi_{1}=-u_{0},
\end{gathered}
$$

where $\Gamma=0$ for model $(a), \Gamma=1$ for model $(b)$, and the dimensionless resistance parameter, $S_{0}$, is defined in Appendix A. Note that system (2.25)-(2.27) is not an eigen-value problem, but a forced system which is driven by the boundary condition (2.27). The equation for the evolution of the sea bed now becomes

$$
\mathrm{i} \omega=\left\langle\left|\tau_{0}\right|^{1 / 2}\left(\frac{3}{2} k \tau_{1}-\lambda k^{2}\right)\right\rangle,
$$

where $\tau_{0}=\partial u_{0} / \partial z$ and $\tau_{1}=\Psi_{1 z z}+v_{1} \partial u_{0} / \partial z$.

Partial differential equation (2.25) can be reduced (approximately) to a system of ordinary differential equations in $z$, using a truncated Fourier series in time which represents the tidal harmonic components. In de Swart \& Zimmerman (1993) and Hulscher et al. (1993), only the first two terms in the series (the residual tide and the sine and cosine parts of the basic tidal motion) were used. In Gerkema (2000), the effects of such truncation were investigated explicitly for sand waves. It was shown that in the regime where the tidal excursion length is much larger than the horizontal spacing between the sand wave crests ( $k R$ large, which is true for our case), the difference between the exact solution and the solution where only the first two timeharmonics were kept, was less than $20 \%$. Also it was pointed out that the qualitative behaviour does not change as a result of the truncation. Due to the assumptions and limitations already made in this study, including higher harmonics would not give better results. Thus in this paper we only keep the first two harmonics, i.e. the solution of equation (2.25) is taken in the form $\Psi_{1}(z, t)=\mathrm{i} \Psi_{10}(z)+\Psi_{1 s}(z) \sin t+\Psi_{1 c}(z) \cos t$. The structure of (2.25)-(2.27) shows that $\Psi_{10}, \Psi_{1 s}$ and $\Psi_{1 c}$ are real functions. The resulting system of ordinary differential equations with boundary conditions is then solved numerically (using the shooting in a fitting point method).

In model $(a)$, the viscosity changes are not taken into account, so $v_{1}$ is identically zero. In model $(b)$, viscosity is given by expression (2.11), and therefore $v_{1}$ is timedependent. Using the known time-components of the near-bed flow, we find the following structure:

$$
v_{1}=\kappa_{0}+\mathrm{i} \kappa_{s} \sin t+\mathrm{i} \kappa_{c} \cos t,
$$


where

$$
\begin{gathered}
\kappa_{0}=-\delta / H+1 /\left.\tilde{u}^{2}\left(u_{s} \Psi_{1 s z}+u_{c} \Psi_{1 c z}\right)\right|_{z=0}, \\
\kappa_{s}=2 /\left.\tilde{u}^{2} u_{s} \Psi_{10 z}\right|_{z=0}, \quad \kappa_{c}=2 /\left.\tilde{u}^{2} u_{c} \Psi_{10 z}\right|_{z=0},
\end{gathered}
$$

and $\tilde{u}$ is defined in equation (2.23). All the velocity values can safely be estimated at $z=0$, since a finite $d$ only makes a contribution at a higher order. As we only consider finite-slip situations, the velocity components at $z=0$ are not zero. Note that equation (2.29) in combination with (2.24) has the form of (2.10) with

$$
\alpha_{1}=\kappa_{0}, \quad \alpha_{2}=-2 /\left.\tilde{u}^{2} \Psi_{10 z}\right|_{z=0}
$$

(this proves property $(\mathrm{v})$ of $\S 2.2$ ). Based on the previously discussed viscosity models, dispersion relation $(2.28)$ can be presented as

$$
\mathrm{i} \omega=\left\langle b\left|\tau_{0}\right|^{b-1} k\left(\Psi_{10 z z}+\left(\kappa_{s} u_{s z} \sin ^{2} t+\kappa_{c} u_{c z} \cos ^{2} t\right)\right)-\lambda\left|\tau_{0}\right|^{b-1} k^{2}\right\rangle_{z=0}
$$

Due to the structure of equation (2.33) and the fact that the function $\Psi_{1}$ is real, it follows that $\operatorname{Re} \omega=0$, i.e. no travelling bed waves are to be expected. This is consistent with the horizontal symmetries of the equations and the choice of the ( $x$-symmetric) basic state. There is no preferred direction in the system. It is often suggested by observations that sand waves travel slowly (see Katoh et al. 1998). Our model does not grasp this effect. In order to model this, one needs to break the horizontal symmetry of the problem (for instance, to introduce a time-independent component in the basic flow or give the bottom a very small slope). This goes beyond the scope of this paper.

\section{Results for a typical offshore location}

We start by presenting some reference values for parameters of the system typical for an offshore North Sea location. The tidal velocity can be taken from 0.5 to $1.5 \mathrm{~m} \mathrm{~s}^{-1}$, the tidal frequency is $\sigma=1.4 \times 10^{-4} \mathrm{~s}^{-1}$. The local depth can vary from $10 \mathrm{~m}$ to about $55 \mathrm{~m}$ (this is the range where sand waves have been observed in the North Sea, see Bijker, Wilkens \& Hulscher 1998). The turbulent viscosity lies between $10^{-3}$ and $10^{-1} \mathrm{~m}^{2} \mathrm{~s}^{-1}$. The slip parameter has a wide interval of values. We experimented with $S_{0}^{\prime}$ from $10^{-4}$ to $10^{-2} \mathrm{~m} \mathrm{~s}^{-1}$ which corresponds to the physically relevant values given in Maas \& van Haren (1987). The typical value for $R$ is several hundred, $H / \delta$ is several units (so that the depth is at most several times the viscous depth) and $k R>1$, which means that the horizontal spacing between the sand wave crests is much smaller than the length of the tidal excursion. Typical values of the parameters in the sediment transport model are given in Appendix B.

Let us take, for example, $H=45 \mathrm{~m}, v_{0}=7 \times 10^{-3} \mathrm{~m}^{2} \mathrm{~s}^{-1}, S_{0}^{\prime}=4.7 \times 10^{-3} \mathrm{~m} \mathrm{~s}^{-1}$ and $U=0.5 \mathrm{~m} \mathrm{~s}^{-1}$. Then,

$$
\delta \approx 10 \mathrm{~m}, \quad H / \delta \approx 4.5, \quad S_{0} \approx 6.7, \Delta T \approx 2 \text { years, }[\tau] \approx 3.5 \times 10^{-4} \mathrm{~m}^{2} \mathrm{~s}^{-2},
$$

and the results are as follows.

Model $(a)$. The result of the linear analysis for model $(a)$ is consistent with what was found by Hulscher (1996). If the currents are very weak, all the modes are damped. As $\lambda$ decreases below some $\lambda_{c}$ (currents become strong enough to overcome gravity), a finite connected bandwidth of excited modes appears between $k=0$ and some finite $k$ (see figure 5 , dotted line, excited modes are between $k=0$ and $k \approx 0.035$ ). The smaller $\lambda$ is, the larger the value of $k$ which corresponds to the maximum of 


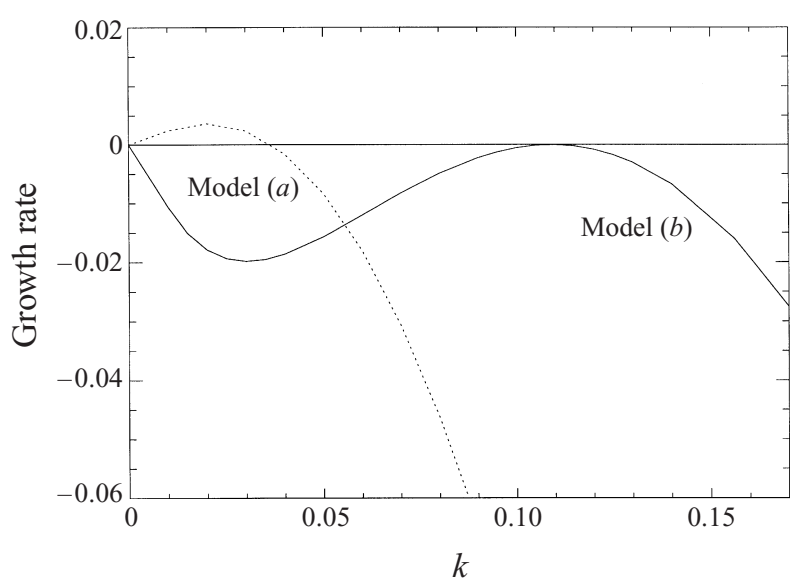

Figure 5. A comparison between the two viscosity models. The dotted line represents model $(a)$, and the solid line model $(b) . \lambda=21.7$ and the rest of the parameters are as in figure 4.
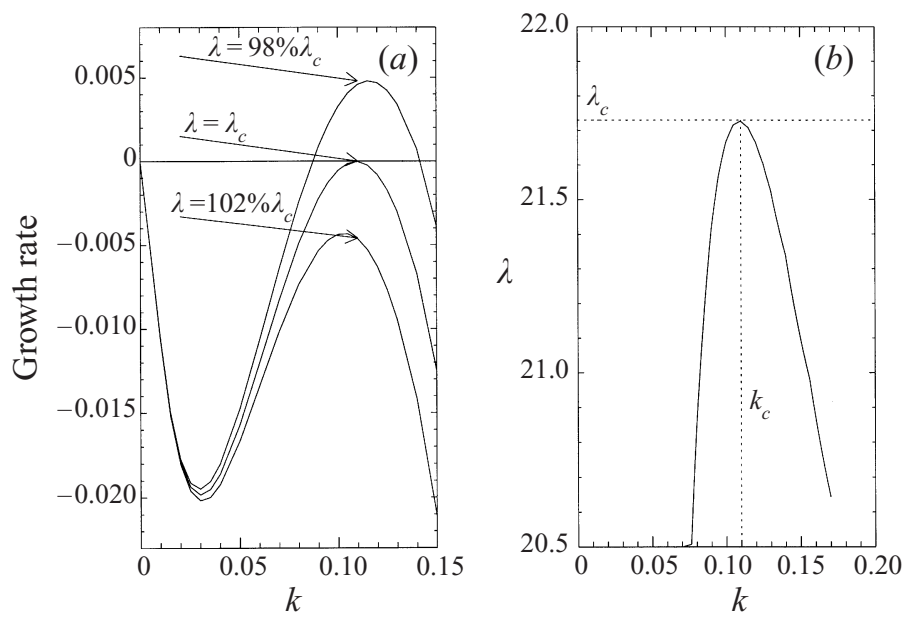

Figure 6. Results of the linear analysis for model $(b), \lambda$ is close to $\lambda_{c}=21.7$. Parameters are the same as in figure $4 . k_{c}$ corresponds to sand waves of length $571 \mathrm{~m}$.

the dispersion curve becomes. However, modes with very small wavenumbers have a positive growth rate for $\lambda<\lambda_{c}$. This is not physical because it predicts the excitation of ultra-long waves.

Model $(b)$. The following parameterization is used: $v_{t}=c_{1} D U\left|u_{n b}\right|_{\text {trunc }}, S^{\prime}=c_{2} U$ $\left|u_{n b}\right|_{\text {trunc }}, c_{1}=1.6 \times 10^{-3}, c_{2}=4.8 \times 10^{-2}$. This leads to the result given by the solid line in figure 5. The corresponding values for $v_{0}$ and $S_{0}^{\prime}$ are the same as given at the beginning of this section, see equation (A 6) and (A 7). The behaviour of the system is then as follows. If the control parameter, $\lambda$, is larger than $\lambda_{c} \approx 21.7$, all the waves are damped due to the gravity term (see figure $6 a$ ). As soon as $\lambda$ becomes smaller than $\lambda_{c}$, an exponentially growing mode appears which corresponds to $k_{c} \approx 0.11$, or in dimensional variables, a wavelength of $571 \mathrm{~m}$. This value is typical for sand waves. The critical value of the control parameter, $\lambda_{c}$, belongs to the physically relevant range given in Appendix B. An important feature is that there is a finite separation 


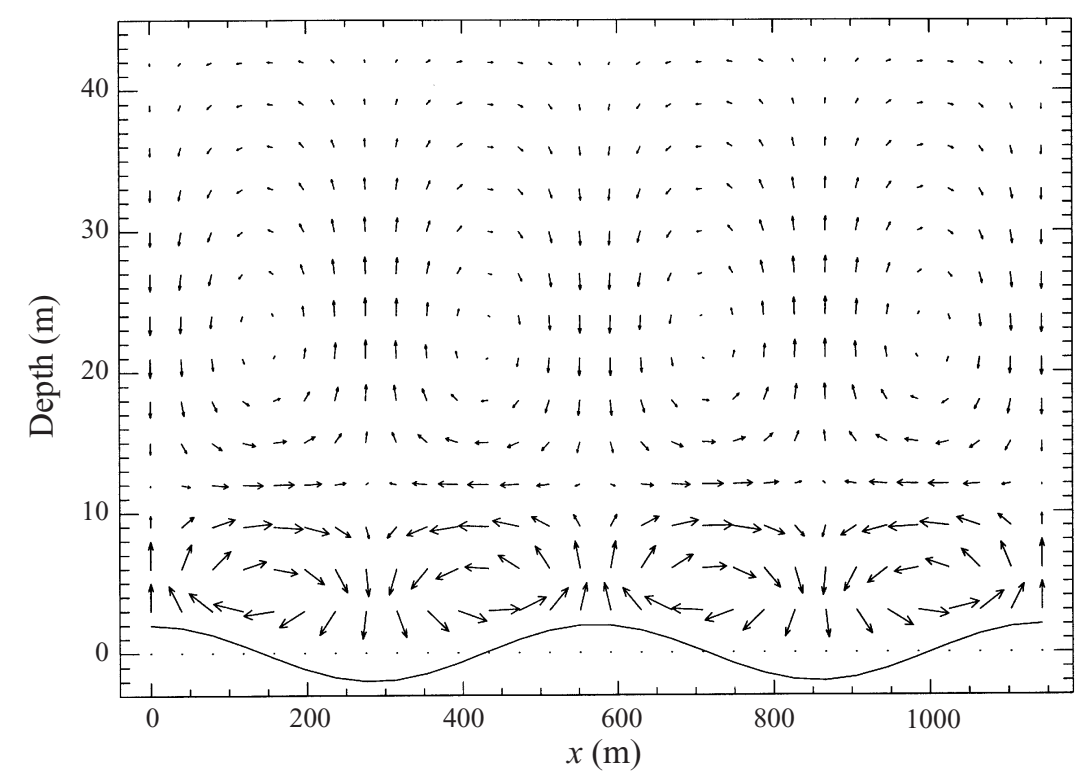

FIGURE 7. The time-independent component of the response flow (comes from $\Psi_{0}$ ) corresponding to the critical condition $\left(k=k_{c}, \lambda=\lambda_{c}\right)$, which looks like two rows of recirculating cells. All the parameters are the same as in figure 4.

between the zero mode and the most excited wave which means that all the very long waves are damped.

An example of the neutral stability curve for model $(b)$ is shown in figure $6(b)$. The time-independent component of the flow solution $\left(u_{1}, w_{1}\right)$ for the critical conditions $(\lambda, k)=\left(\lambda_{c}, k_{c}\right)$, is presented in figure 7 . The time-independent flow looks like two rows of circulating cells. The flow near the bottom is directed up the hill. This result is predicted numerically, but it is very hard to observe in nature, because the residual currents are very weak. For a wave as high as $10 \%$ of the undisturbed water depth, and the tidal amplitude of $0.5 \mathrm{~m} \mathrm{~s}^{-1}$, the amplitude of the horizontal velocity perturbation, $\Psi_{0 z}$, is only about $0.5 \mathrm{~cm} \mathrm{~s}^{-1}$ which is beyond the accuracy of measurements at the present time.

Finally, we can evaluate the coefficients $\alpha_{1,2}$ of representation (2.10) using expression (2.32). Computations show that $\alpha_{1}$ is always negative and tends to a negative constant when $k \rightarrow 0$. Note that the expression for $\alpha_{1}$ consists of two parts: the first term in expression (2.30) comes from the direct dependence of $v_{t}$ on depth, $D$, and the second term is the contribution from the $u_{n b}$-dependence of $v_{t}$. Both contributions are negative, but the latter is much larger. This means that the effect of viscosity changes due to the direct $D$-dependence is too small to make a difference, and only the $u_{n b}$ dependence of the viscosity is important for the flow dynamics (see also the comment in the summary of $\S 4$ ). The coefficient $\alpha_{2}$ is proportional to $k$ for small values of $k$. Its sign is determined by the sense of rotation of the time-independent cells. For small values of $k$, the cells move in such a way that near the bed the flow is directed from crests to troughs, and then $\alpha_{2}$ is positive. As the direction of rotation changes, $\alpha_{2}$ becomes negative. In figure 8 , we show qualitatively the viscosity distribution corresponding to $k=k_{c}$. It follows that the boundary layer, $\sqrt{2 v_{t} / \sigma}$, becomes thicker on the upstream slopes of sand waves and thinner on the downstream slopes, i.e. there 


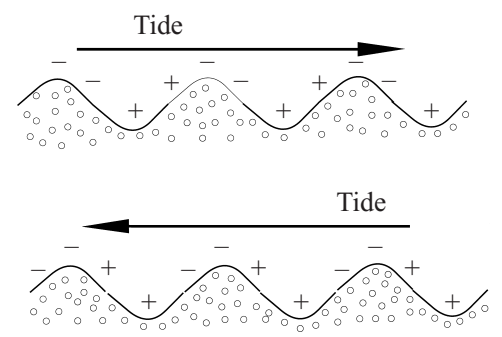

FIGURE 8 . The viscosity changes corresponding to $k=k_{c}$ (i.e. $\alpha_{1}<0, \alpha_{2}<0$ ). Here, $+(-)$ denotes an increase (decrease) in $v_{t}(x, t)$.
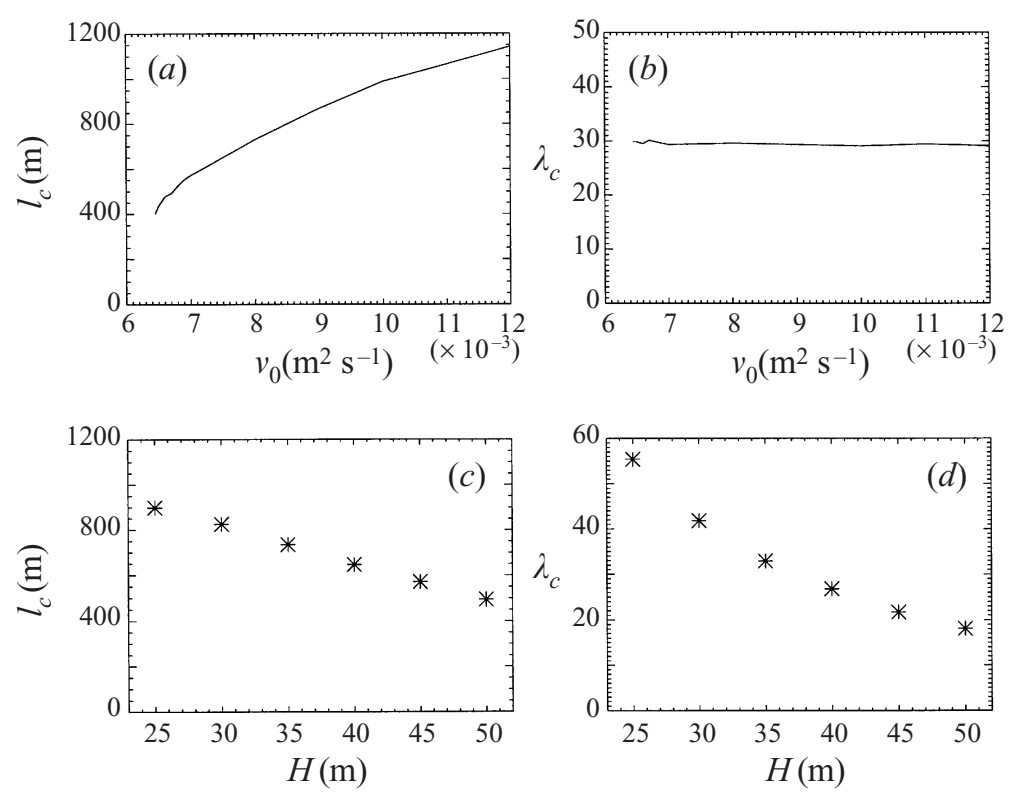

FIGURE 9. The $v_{0}$ and $H$-dependence of the critical wavelength, $l_{c}$, and the critical control parameter, $\lambda_{c}$. For $(a, b)$, parameter $H=45 \mathrm{~m}$.

is always less turbulence behind each bump. On the average, the turbulent boundary layer is thicker in troughs than it is over crests.

\subsection{Parameter dependence of the results}

The same qualitative picture (the shape of the dispersion relation and the flow field) holds for a large range of physically relevant parameters, giving slightly different critical wavenumbers and different critical values for the control parameter. This is an indication of the fact that the mechanism is quite generic. We have performed some calculations to determine the dependence of the most excited wavelength (and also the critical value of the control parameter, $\lambda_{c}$ ) on physical parameters of the system. The results are presented in figure 9. Plots $(a)$ and $(b)$ show the viscosity dependence of the most excited wavelength, $l_{c}=2 \pi / k_{c}$, and $\lambda_{c}$. There, the constant in the $S^{\prime}$ parameterization is fixed to be $c_{2}=4.8 \times 10^{-2}$, and $c_{1}$ changes to give different values for the viscosity, $v_{0}$. As the near-bed velocity changes, the value of $S_{0}^{\prime}$ changes slightly as well, which is indicated in table $2(a)$. The resulting plot shows that the wavelength of sand waves grows with the eddy viscosity. In fact, the functional 
(a)

$\begin{array}{llllllll}v_{0} & 0.6 & 0.7 & 0.8 & 0.9 & 1.0 & 1.1 & 1.2\end{array}$

$\begin{array}{lllllllll}S_{0}^{\prime} & 4.5 & 4.7 & 4.9 & 5.0 & 5.1 & 5.3 & 5.4\end{array}$ $(b)$

$\begin{array}{ccccccc}H & 25.0 & 30.0 & 35.0 & 40.0 & 45.0 & 50.0 \\ v_{0} & 3.3 & 4.2 & 5.1 & 6.0 & 7.0 & 8.0 \\ S_{0}^{\prime} & 4.0 & 4.2 & 4.4 & 4.6 & 4.7 & 4.8\end{array}$

TABLE 2. (a) Viscosity and resistance parameter values for figures $9(a)$ and $9(b)$. $(b)$ The depth dependence of the viscosity and the resistance parameter for figures $9(c)$ and $9(d) . H$ is given in $\mathrm{m}$, $v_{0}$ in $10^{-2} \mathrm{~m}^{2} \mathrm{~s}^{-1}, S_{0}^{\prime}$ in $10^{-3} \mathrm{~m} \mathrm{~s}^{-1}$.

dependence of $l_{c}$ is best described by $l_{c} \propto \sqrt{v_{0}}$ which indicates that the horizontal wavelength scales with the turbulent boundary layer thickness, $\delta$. This also coincides with the observation of Hulscher (1996) that the horizontal size of sand waves grows with viscosity. On the other hand, the critical control parameter dependence on the viscosity is very weak (see figure $9 b$ ). The values found for $\lambda_{c}$ fall in the physically relevant range.

Figures $9(c)$ and $9(d)$ show the depth dependence of $l_{c}$ and $\lambda_{c}$. The constants used in the viscosity and resistance parameterization are $c_{1}=1.6 \times 10^{-3}$ and $c_{2}=4.8 \times 10^{-2}$, so that $v_{0}$ and $S_{0}^{\prime}$ change with the depth according to table 2(b). Figure 9(c) shows that the wavelength of sand waves decays with depth even though the dependence on $H$ is not too strong. Note that in the present analysis, parameters of the system such as the tidal amplitude and the exponent $b$ (equation (2.9)) were kept constant even though the depth was varied. In a real system, those parameters might change with depth which in turn can influence the depth dependence of $l_{c}$. Therefore, the results in figure $9(c)$ cannot be compared with measurements directly. As shown in figure $9(d)$, the critical control parameter decays significantly with depth. This means that one can expect to have $\lambda<\lambda_{c}$ with a higher probability in a shallower sea than in a deeper sea (for most situations, $\lambda<330$, see Appendix B). In other words, sand waves are more likely to be generated in a shallower sea than they are in a deeper sea. This is in agreement with the observation of Wilkens (1997) which indicates that in the North Sea sand waves do not appear at all if the depth is larger than about $55 \mathrm{~m}$.

\subsection{An analytical estimate for the sand wave wavelength}

The length scale of the most favourable wave depends on the turbulent boundary layer thickness which is in turn defined by the tidal frequency and the eddy viscosity. The result for depth dependence suggests that the wavelength slightly decreases with $H$. This has an explanation based on the main balance which determines the first excited wavelength, namely, the balance between gravity and the flow strength. To obtain a rough estimate, we can assume that the tidal response (the horizontal component of the residual current) is proportional to the $z$-derivative of the tidal flow times the amplitude of the perturbation, $\hat{h}$. This is because the term $u_{0} \hat{h}$ is the main driving term in the linearized hydrodynamical system for $\Psi_{1}$, see equation (2.27). Therefore, we can write $\hat{h} \Psi_{1} \sim \hat{h} u_{0}$. Note that this exactly satisfies equation (2.27) with $\Gamma=0$ (here we neglect the weak effect of the direct depth-dependence of the viscosity). Now, we can substitute this estimate for the response current into the linearized equation (2.9). Considering the balance between the scraping term and the down-hill term, we find

$$
v_{0} u_{0 z z} h \sim \lambda^{\prime} k h,
$$


where the perturbation of the bottom shear stress is $\tau_{1} \sim v_{0} \Psi_{1 z z} \sim v_{0} u_{0 z} \delta^{-1} \sim \tau_{0} \delta^{-1}$. Now, substituting $v_{0} u_{0 z z} \sim \tau_{0} / \delta$ and using $\lambda^{\prime} /[\tau]=\lambda$, we get $k \sim 1 /(\lambda \delta)$. Reversing this expression and taking the critical values, we derive the simple estimate

$$
l_{c} \sim \lambda_{c} \delta,
$$

i.e. the wavelength of the sand waves is (usually more than) ten times larger than the turbulent boundary layer thickness. This is indeed a correct estimate and it coincides with the numerical fact that $l_{c}$ scales with $\sqrt{v_{0}}$. Also, since $\lambda_{c}$ decays with depth, $l_{c}$ decreases with depth as well, as indicated in figure $9(c)$.

\section{Mechanisms for sand wave excitation}

In this section we explain why viscosity model $(b)$ is successful in suppressing ultra-long waves, whereas model $(a)$ is not. Note that in order to uncover all the necessary physics, one does not need to use the concrete functional form (2.11) of the viscosity parameterization. We will show that if the linearized viscosity functional is given by (2.10) with $\alpha_{1}<0$ for small $k$ (property ( $\mathrm{v}$ ) of $\S 2.2$ ), this provides a damping mechanism for ultra-long waves. To start, we notice that if a perturbation is added to the sandy bed, then the flow changes due to two forcing mechanisms: $(b))$;

(a) directly, due to the bed perturbations (this happens both in models $(a)$ and

(b) indirectly, because the bottom perturbations induce viscosity changes as in equation (2.10) (this is only the case for model $(b)$ ).

When studying model $(b)$, it is instructive to separate the driving term coming from the depth- and velocity-dependent viscosity (equation (2.11)), neglecting the direct effect of the bumps on the flow. Note that the $x$-dependent viscosity changes alone do not correspond to any physically relevant situation. However, this approach enables one to single out the role of viscosity perturbations.

Below we present some (numerically) observed properties of the response flow and give qualitative arguments to explain them. From the two main properties, all the important details of the instability and damping mechanisms can be understood.

Property 1. For model (a), periodic bed perturbations produce a time-independent component of the response flow directed as in figure 10.

Remark. The flow dynamics described here has been observed by other authors (Lyne 1971; Blondeaux 1990). It can be explained by looking at pressure gradients induced by the bed geometry. However, we choose to develop another argument based on the flow geometry, because this argument can be extended to the case of viscosity perturbations.

We start with a horizontal tidal motion over a flat bed. The dotted lines in figure 11(a) depict the profile given by equation (A 8), i.e. the solution of problem (A 1)-(A 5). Then we add a periodic perturbation to the bottom and assume that its wavelength is very large in comparison with $H / \delta$. We first consider a tide in the positive direction.

The tidal flow profile has to adjust to the changed bed geometry, as the flow aims to satisfy the bed boundary condition and the water mass conservation law. The solid lines in figure 11 $(a)$ represent the perturbed flow. They can be thought of as solutions of problem (A 1)-(A 5), with the bottom boundary condition, (A 3), evaluated at $z= \pm \hat{h}$ instead of $z=0$. At a trough, the excess tidal velocity is positive near the bed, and negative in the upper part of the water column (since the area 
(a) Small $k$

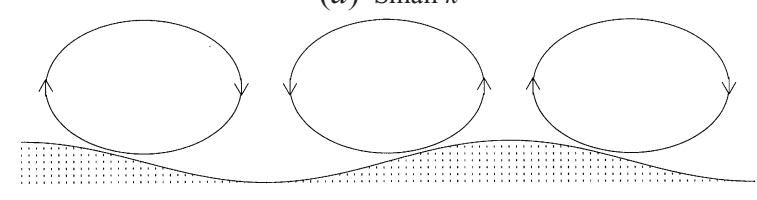

(b) Large $k$

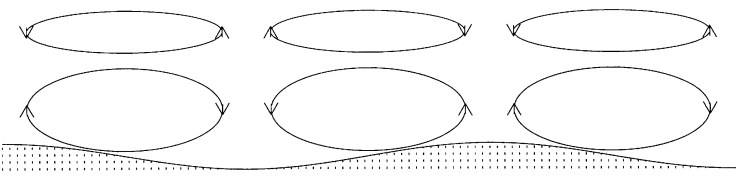

FIGURE 10. The geometry of the time-independent component of the response flow for the constantviscosity model. (a) For small $k$, there is a single row of cells encouraging the bed perturbation. (b) As $k$ grows, a second row of cells appears on top. The lower row keeps its orientation.

(a)

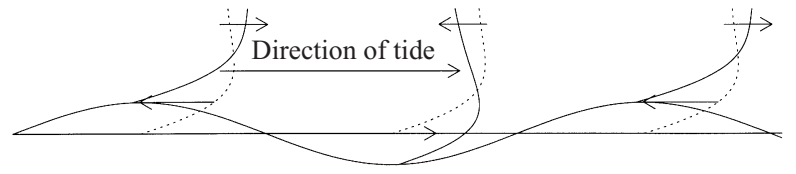

(b)

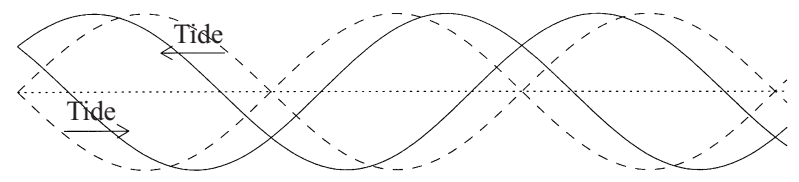

(c)

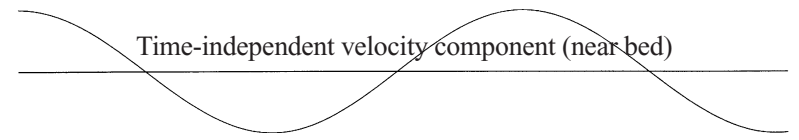

FIGURE 11 . The instability mechanism. (a) Once the flat bed is perturbed, the $M_{2}$-tide (dotted line) has to adjust (solid line) so that the near-bed flow becomes smaller near crests and larger near troughs. (b) The near-bed flow response for both directions of the tide is shown for a no-inertia case (dashed lines) and in the presence of viscosity (solid lines). Due to viscosity, the flow response lags behind. $(c)$ The net effect after a full tidal period at a fixed (near-bed) level. The flow has a negative sign (directed to the left) near the left-hand side of the trough and it is positive (directed to the right) near the right-hand side of the trough.

under the profile cannot change due to the water mass conservation). The excess flow has the opposite sign above a crest. The excess flow for some $z$ near the bed is shown in figure 11(b), dashed line.

Now, if there were no viscosity in the system, this forward tidal motion would be exactly cancelled by the backward motion (figure $11 b$, the dashed lines). However, in a real physical system there is inertia due to viscosity, i.e. the changes in the flow profile which start at the bed need some time (and consequently some horizontal distance $x$ ) to be transferred upwards. As a result, there is a $z$-dependent lag in the flow response to the bottom changes, so that for any finite $z$, the maximum of the response is shifted in the positive direction when the tide is moving to the right and in the negative direction in the backward motion (see figure $11 b$, solid line). In short, the response always lags behind. Graphically, the retarded flow response is shown in figure 11(b,c). 
(a) Small $k$

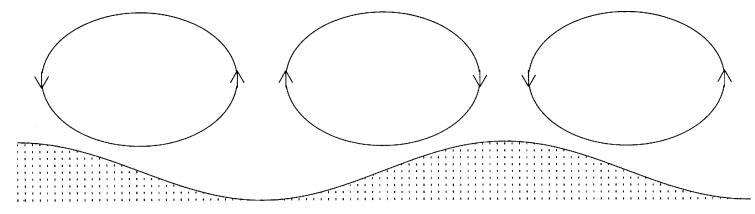

(b) Large $k$

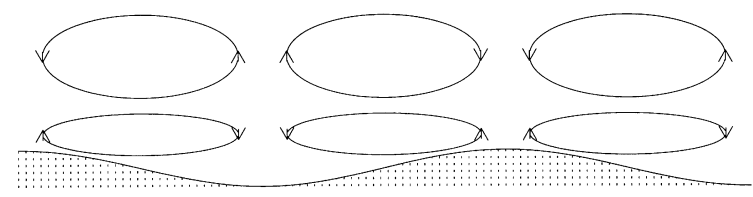

FIGURE 12. The geometry of the time-independent component of the response flow in the (artificial) case when the direct influence of bed perturbations is not taken into account and the viscosity is perturbed. (a) For small $k$, there is a single row of cells destroying the bed perturbation. (b) As $k$ grows, a second row of cells appears from below. The direction of the near-bed flow reverses. Now the bed perturbation is encouraged.

The resulting effect after one tidal period is found from the superposition of the two solid lines in figure $11(b)$ and gives a non-zero residue component of the velocity (figure 11c). This component does not depend on time and has indeed the correct sign: the circulating cell is oriented as in figure 10. It is clear that such cells will carry sediment from troughs to crests and have a (weak) reverse flow in the upper part of the water column.

In agreement with the above argument, it was observed that the residual flow looks like one row of vortices (see figure 10a). The direction of the flow near the bed is the same (towards the crests) for all values of $k$ within the validity of the model. However, when $k$ increases (and the water is deep enough), a new row of cells can appear on the top of the first one, as shown in figure $10(b)$. Note that for the physical parameters given in $\S 3$, the generation of a second row of vortices was not observed for wavenumbers within the validity of the model. Only when the depth was taken as large as $67 \mathrm{~m}$, did the new vortices near the surface appear, starting with some $k$.

PROPERTY 2. Let us ignore the direct influence of the bed perturbations. If the value of the viscosity coefficient is smaller near the crests and larger near the valleys (i.e. $\alpha_{1}<0$ in (2.10)), the time-independent component of the flow response is directed as in figure 12 .

We use the same graphical method as we did when studying the effects of a bed perturbation. By analogy with figure 11(a), the dotted lines in figure 13 again are the solution of problem (A 1)-(A 5). The solid lines represent the flow profile when the viscosity varies horizontally (the vertical profile gradients near the bottom are larger where the viscosity is smaller and smaller where the viscosity is larger). Comparing the response flow in figure 13 with the corresponding flow in figure 11, we can see that they have opposite directions. Therefore, the flow induced by viscosity perturbations looks like recirculating cells directed from crests to troughs of sand waves.

This is not surprising if one thinks of the flows corresponding to the varied viscosity as solutions of problem (A 1)-(A 5) with $v_{t}=v_{0} \pm \hat{h} v_{1}$, i.e. with $\delta$ being smaller on tops of crests and larger in the valleys. Note that $\delta$ only enters the problem as the 


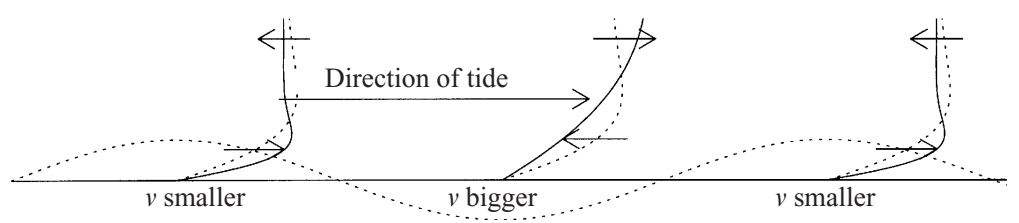

FIGURE 13. The changes in the flow induced indirectly, through a depth-dependent viscosity. The direct influence of bed perturbations is not shown.

ratio $H / \delta$, i.e. the surface boundary condition is now evaluated at a larger height over tops of crests and at a smaller height in the valleys. Therefore, the changes of viscosity counteract the changes of depth. Namely, where the bed is lifted by the amount $\hat{h}$ (depth decreased), the viscosity becomes smaller and the relative depth, $H / \delta$, increases! So effectively, the changes of viscosity reduce the changes in the relative depth caused by the bottom perturbation.

We have shown that for small values of $k$, the time-independent flow looks like one row of vortices, see figure 12(a). However, if $k$ increases and exceeds some value $\tilde{k}_{0}$, a second row of cells appears from below and this flow rotates in the opposite direction to the initial cells (see figure 12b). The initial cells move towards the surface. Therefore, for $k<\tilde{k}_{0}$, the viscosity changes give rise to a flow which has a tendency to flatten the sand waves, i.e. the viscosity changes have an effect opposite to the effect of the bed perturbation. For $k>\tilde{k}_{0}$, the effect of perturbed viscosity enhances the effect of bottom perturbations. This can be explained in the following way. As it was argued, viscosity perturbations effectively act like surface perturbations, which means that near the surface the direction of the secondary flow always stays the same. As in the case of bed perturbations, as $k$ grows, the flow has a tendency to separate, and instead of one row of vortices there are two. In the case of bed perturbations, the new row of cells appeared from the surface. In the case of viscosity perturbations, it is at the bottom where the new row of cells appears. The initial cells persist, so the flow away from the bottom has the same direction as before, and the direction near the bottom changes sign. The value of $\tilde{k}_{0}$ is usually such that the corresponding wavelength is of order of (or smaller than) the tidal excursion length (equal to $2 \pi U / \sigma$ ), i.e. this effect belongs to the range of length scales where the model holds.

Remark. Note that the vortices of figure 12(a) immediately lead to an exponential decay of the underlying bed perturbation and therefore can only be observed numerically.

Summary. As we have seen, Property 1 generates sand waves, and Property 2 provides a mechanism for damping ultra-long waves. The relative strength of the two effects depends on the concrete model. In the previous section we showed that for model (2.11), the time-independent component of the viscosity perturbation satisfies the assumption of Property $2\left(\alpha_{1}<0\right)$, and the time-dependent component is small for small values of $k$. This means that the flow has both Properties 1 and 2 and the flow can be thought of as a superposition of the two types of flow. For small values of $k$, the time-independent flow component consists of the contributions of figures 10(a) (direct instability mechanism) and 12(a) (the flow of Property 2). It was demonstrated numerically that the effect of Property 2 is noticeable, i.e. the vortices of figure $12(a)$ are strong enough to reverse the sense of rotation of the flow cells. Figure 6(a) shows that this effect creates a finite gap between the zero mode and the most excited mode. Numerical simulations of the previous section showed that it is the near-bed velocity 
dependence of the viscosity which is responsible for damping modes with small $k$. The direct $D$-dependence does not play a significant role. For larger values of $k$, the cells of Property 2 reverse their direction of rotation. The time-independent flow corresponding to the first excited wavenumber, shown in figure 7, is a superposition of flows given in figures $10(a)$ and $12(b)$.

\subsection{Morphodynamics}

As we know the bottom shear stress behaviour for models $(a)$ and $(b)$, we can now comment on the combined effect of gravity and hydrodynamics which manifests itself in the dispersion relation. From equation (2.33) we can see that the growth of sand patterns is determined by the balance of the two terms:

$$
\mathrm{i} \omega \propto\left(\left.k \Psi_{10 z z}\right|_{z=0}(k)-\text { const } k^{2}\right),
$$

the 'scraping term' and the term representing gravity.

In model $(a)$, for every $k>0$ there is a sand wave amplifying circulation near the bed. In this case, $\Psi_{10 z z}$ at $z=0$ is always positive, grows linearly for small values of $k$ and then reaches saturation (grows slower than $k$ for large $k$ ). Therefore, from equation (4.1) it is clear that for large $k, \operatorname{Re} i \omega<0$. These short bed waves are damped because of the gravity term. The long waves are either damped for (very) large values of the control parameter $\lambda$, or excited as the stress parameter passes its critical value. When $\lambda$ becomes smaller than critical, the first waves that get excited are ultra-long waves, which is not physical.

A different behaviour is observed for viscosity model $(b)$. There, viscosity perturbations lead to the decay of long waves and encourage the growth of shorter waves. In dispersion relation (4.1), the term $k \Psi_{10 z z}(z=0)$ is now negative for small $k$ and changes sign as $k$ becomes larger. The morphodynamics of the system is now governed by three main effects:

growth of bed features due to the direct flow response to the bed perturbations;

damping of short bed waves due to the downhill effect in the sediment transport formula (an effect of gravity);

damping of ultra-long waves due to the $u_{n b}$-dependence of turbulent viscosity (a hydrodynamical effect).

Note that the damping of short waves is not only due to the gravity, but also (and mainly) because the flow itself acts to move the sand from the crests down to the troughs. The balance of the three effects leads to the choice of the most unstable wavenumber.

\section{Discussion and conclusions}

In this paper it was shown that if parameterization $(b)$ is employed for the eddy viscosity, the system chooses a most excited mode whose wavelength turns out to be similar to the observed sand wave spacing.

We will now discuss the applicability and limitations of the model used in this work. The shallow water approximation was used in order to make the equations simpler. It was checked that the mechanisms and results do not change if one drops this approximation. It was also checked that including the effects of the horizontal turbulent viscosity, $v_{t}^{\text {hor }}$, did not make a significant difference.

Next, it must be noted that not all horizontal scales can be studied by means of our model. Namely, hydrodynamical equations (2.18)-(2.21) can only be used for horizontal distances between about $100 \mathrm{~m}$ and $80 \mathrm{~km}$. The upper bound comes from 
condition $k R>1$, i.e. only distances smaller than the characteristic tidal wavelength (which is about $800 \mathrm{~km}$ ) can be considered. However, one should note that Coriolis effects (which becomes important at horizontal scales of about $5 \mathrm{~km}$ ) are not included in this paper. The lower bound states that the horizontal distances must be bigger than the turbulent boundary layer thickness (the processes that take place on smaller scales are averaged out and included in the turbulent viscosity parameter). Note that the range of horizontal scales allowed by this model covers the range of sand wavelengths well.

Related studies (e.g. Blondeaux 1990 and, recently, Gerkema 1998) have shown that the ratio $H / \delta$ is an important parameter. The value of $H / \delta$ determines whether or not the constant-viscosity parameterization leads to a damping of the very long waves. In the case when $H / \delta \gg 1$ (sand ripples), the constant-viscosity model leads to the damping of the ultra-long waves. In this case, this is indeed the only applicable model since the viscosity there is just the molecular viscosity. The damping of the long waves can be explained if one uses the series $\Psi_{1}(k)=c_{1} k+c_{2} k^{2}+\cdots$. The first constant, $c_{1}$, turns out to be inversely proportional to the ratio $H / \delta$ and therefore it disappears in the deep water limit. This result can be proven analytically for small values of $k R$ if one repeats the analysis of Lyne (1971) in the case of a finite depth, and the same (linear) dependence of $c_{1}$ on $\delta / H$ was found numerically for large values of $k R$ (this is in agreement with Gerkema 1998). As a consequence, in the case of deep water, the recirculating cells which tend to build up the sand bumps are quadratically weak when $k$ is small, and the long waves are naturally damped by the gravity term (which is quadratic, and if the multiplier $\lambda$ in front of it is large enough, the resulting growth rate is negative). In the case of sand waves, when $H / \delta \sim 1$, the recirculating cells grow linearly with $k$ and are too strong to be overcome by gravity. This shows that the simple model where the turbulent viscosity is assumed to be a constant fails to grasp the important physical process of damping long bed waves. In this paper we have presented a model where the assumption that the eddy viscosity is a constant was dropped. It was demonstrated that this gives a way to overcome the shortcoming of the previous model.

We would like to emphasize again that the model we used is not an artificial way to suppress long waves in the system. The notion of a Stokes layer is only well-defined in systems with a laminar flow where $v$ is the molecular viscosity. In a laminar flow, the friction effects are described well by this constant. However, it is only by analogy that a Stokes layer is introduced in systems with a well-developed turbulence, and it does not follow that this layer thickness should be a constant quantity. In fact, this thickness must change if the velocity profile and the depth change horizontally. In this work we used a very simple phenomenological viscosity model which could be refined in many different ways. One can for instance let the viscosity be a $z$ dependent quantity, or use another way to estimate the characteristic flow velocity which enters the expression for the eddy viscosity. The concrete functional form of viscosity parameterization is not necessary to grasp the effect of damping ultra-long waves. A general conclusion that follows from the present analysis is the following: if (for small values $k$ ) the viscosity is larger in troughs than it is over crests, this provides a mechanism for suppressing long waves.

Another important result is that the wavelength of the most excited waves can be estimated using a very simple formula, equation (3.3). Using this relation, we can conclude that $\delta$ plays a crucial role in the dynamics. This can be extended to other models which include a similar sediment transport formula and have an appropriate control parameter. For instance, in the case of sea waves, the Stokes layer thickness 
is very small, and the resulting periodic features (sand ripples) have a wavelength of several centimetres (Blondeaux 1990).

This paper is devoted to the linear analysis of the model and the uncovering of basic physical mechanisms responsible for the sand wave excitation. The next step is to predict a longer-scale time and space behaviour of sand waves. This can be done by means of a nonlinear analysis. The result of the present paper provides a good starting point for developing a weakly nonlinear theory of sand waves. Some forthcoming work will give more evidence that the model proposed in this paper indeed leads to qualitatively correct results when one tries to estimate the height of the saturated nonlinear sand waves (see Komarova \& Newell 2000).

This paper is based on work in the PACE-project, in the framework of the EUsponsored Marine Science and Technology Program (MAST-III), under contract no. MAS3-CT95-0002. This work was co-sponsored (S. H.) by the Technology Foundation STW, contract TCT.4466. One of the authors (N. K.) thanks the Department of Civil Engineering, University of Twente for their hospitality during her stay there while working on the paper.

\section{Appendix A}

The system of equations that the basic tidal solution satisfies can be written as

$$
\begin{aligned}
& \left(2 \partial_{t}-\partial_{z}^{2}\right) u_{0 z}=0 \\
z=H / \delta: & u_{0 z}=0, \\
z=0: & u_{0 z}=S^{\prime} \delta / v_{t} u_{0}=S_{0}^{\prime} \delta / v_{0} u_{0} \equiv S_{0} u_{0},
\end{aligned}
$$

where $S_{0}^{\prime}$ is the resistance parameter, $S^{\prime}$, corresponding to the basic tide, and $S_{0}$ is the dimensionless resistance parameter. Note that this system allows many different solutions. We want the basic solution to be an $M_{2}$-tide; therefore we should prescribe the time-dependence of $u_{0}$ to be

$$
u_{0}(z, t)=\tilde{u}(z) \sin (t+\Phi(z)) .
$$

This reduces the above partial differential equation to a system of ordinary differential equations in $z$ for functions $\tilde{u}$ and $\Phi(z)$. The resulting system still does not have a unique solution. If we impose a normalization condition,

$$
(\delta / H) \int_{0}^{H / \delta} u_{0}(z, t) \mathrm{d} z=\sin t,
$$

the solution $u_{0}$ can be found in a unique way. Condition (A 5) is used in order to fix the vertically averaged flow (in dimensional variables) to be $U \sin \sigma t$. Finally, we need to define the parameters in equations (A 1)-(A 3). We have the following definitions:

$$
\begin{gathered}
\delta=\sqrt{2 v_{0} / \sigma}, \quad v_{0}=\left\{\begin{array}{l}
\operatorname{model}(a): \text { a given constant } \\
\operatorname{model}(b): c_{1} H U \tilde{u}(z=d) / \sqrt{2},
\end{array}\right. \\
S_{0}=S_{0}^{\prime} \delta / v_{0}, \quad S_{0}^{\prime}=\left\{\begin{array}{l}
\operatorname{model}(a): \text { a given constant } \\
\operatorname{model}(b): c_{2} U \tilde{u}(z=d) / \sqrt{2} .
\end{array}\right.
\end{gathered}
$$

Expressions (A 6)-(A 7) define the viscosity and the resistance parameter. In model $(a)$, they are external constants of the system, whereas in model $(b)$ they depend on 
the unknown function $u_{0}$. It is important to note that due to the structure of the basic state (A 4), even in the case of model $(b), v_{0}$ and $S_{0}^{\prime}$ are time-independent quantities. This is a consequence of the truncation in definition (2.11). To make this more clear, we now explicitly derive equation $v_{0}=c_{1} H U \tilde{u}(z=d) / \sqrt{2}$.

We start with definition (2.11) and use the basic tidal solution (in the form (A 4)) to find the near-bed velocity, $u_{n b}=u_{0}(z=d)$. Next, we expand the absolute value of this expression into a Fourier series in time, $\left|u_{n b}\right|=\tilde{u}(z=d) / \sqrt{2}+u^{(2)} \sin \left(2 t+\Phi^{(2)}\right)+\cdots$. It contains time-independent harmonics but it does not contain a term reflecting the tidal frequency, i.e. $\propto \sin \left(t+\Phi^{(1)}\right)$, which is a consequence of the specific timebehaviour of the $M_{2}$-tide and as well as the fact that we are taking an absolute value of this function. Finally, in order to satisfy the requirement that the time-dependence of $v$ cannot contain higher harmonics than the ones present in the flow, we need to neglect all the time harmonics except for the zeroth and the first one. Therefore, only the first term in the expansion for $\left|u_{n b}\right|$ should be taken into account. From this the time-independent expressions (A 6)-(A 7) follow.

Equations (A 1)-(A 7) are a self-consistent system for determining the basic tidal solution, $u_{0}$, together with the corresponding turbulent viscosity, $v_{0}$. For both viscosity models, this system can be solved exactly:

$$
\begin{aligned}
u_{0} & =\frac{\mathrm{i} H}{2 \delta} \frac{\cosh (1+\mathrm{i})(H / \delta-z)-\cosh (1+\mathrm{i}) H / \delta-(1+\mathrm{i}) / S_{0} \sinh (1+\mathrm{i}) H / \delta}{\left(H / \delta(1+\mathrm{i}) / S_{0}-(1+\mathrm{i})^{-1}\right) \sinh (1+\mathrm{i}) H / \delta+H / \delta \cosh (1+\mathrm{i}) H / \delta} \mathrm{e}^{\mathrm{i} t}+\text { c.c. } \\
& \equiv u_{s}(z) \sin t+u_{c}(z) \cos t .
\end{aligned}
$$

Solution (A 8) is similar to the ones obtained in Gerkema (2000). A more general (three-dimensional) version of this solution was obtained, for instance, in Visser et al. (1994) and Hulscher (1996). In the limit of the infinite depth, it becomes exactly the solution used by Blondeaux (1990). Note that solution (A 8) holds for each of the viscosity models.

\section{Appendix B}

In this Appendix, a bed-load sediment transport model will be derived, which accounts for both direct bed-slope effects and the influence of the bed slope on the threshold of motion.

Following Fredsoe \& Deigaard (1992, p. 206), the volumetric sediment transport, $q$, (measured in $\left.\mathrm{m}^{2} \mathrm{~s}^{-1}\right)$ is given by

$$
q=\Phi_{b} \sqrt{(s-1) g d^{3}}
$$

in which $\Phi_{b}$ is non-dimensional transport, $s=\rho_{s} / \rho$ is the relative density of the sediment $\left(\rho_{s}\right)$ with respect to water density $(\rho), g$ is the acceleration due to gravity $\left(\mathrm{m} \mathrm{s}^{-2}\right)$ and $d$ is grain diameter $(\mathrm{m})$. To make the Meyer-Peter-Muller formula (Fredsoe \& Deigaard 1992, p. 214) work for tidal motion (instead of a unidirectional flow), the Heaviside function, $\mathscr{H}$, is introduced. It ensures no motion below the threshold. If $\Theta^{\prime}$ denotes the (non-dimensional) Shields parameter and $\Theta_{c}$ is the critical Shields parameter above which sediment starts to move, the transport is given by

$$
\Phi_{b}=8 B|| \Theta^{\prime}\left|-\Theta_{c} t\right|^{3 / 2} \Theta^{\prime} /\left|\Theta^{\prime}\right| \mathscr{H}\left(\left|\Theta^{\prime}\right|-\Theta_{c}\right) .
$$

Note that this formula correctly models the sign of the sediment transport. $\Theta^{\prime}$ is 
related to the volumetric bed shear stress, $\tau_{b}$ (in units $\mathrm{m}^{2} \mathrm{~s}^{-2}$ ), by

$$
\Theta^{\prime}=\frac{\tau_{b}}{g(s-1) d} .
$$

Equation (B 2) includes two effects. One is the existence of a critical $\Theta_{c}^{\prime}$ which gives the threshold of motion. The other is contained in the factor $B$ and describes the fact that the slope directly changes the sediment transport (Bagnold 1956). Both $\Theta_{c}^{\prime}$ and $B$ depend on the angle $\phi$, which is given by the slope gradient, $-h_{x}$. We have (Fredsoe \& Deigaard 1992, p. 205)

$$
\Theta_{c}=\Theta_{c 0} \cos \phi\left(1-\frac{\tan \phi}{\tan \phi_{s}}\right)=\Theta_{c 0} \frac{1}{\sqrt{1+h_{x}^{2}}} \sqrt{1+\frac{h_{x}}{\tan \phi_{s}}},
$$

in which $\phi_{s}$ is the friction angle, the angle of no repose. The second effect of the sloping bed can be expressed as

$$
B=\left(1-\tan \phi / \tan \phi_{s}\right)^{-1}=\left(1+h_{x} / \tan \phi_{s}\right)^{-1} .
$$

Substituting equations (B2)-(B 5) into (B1), we find the sediment transport as a function of the volumetric shear stress, $\tau_{b}$, and bed gradient, $h_{x}$ :

$$
q=C_{1} \frac{\tau_{b}}{\left|\tau_{b}\right|}\left|\tau_{b}-C_{2}\left(\frac{1+h_{x} / \tan \phi_{s}}{1+h_{x}^{2}}\right)\right|^{3 / 2}\left(1+\frac{h_{x}}{\tan \phi_{s}}\right)^{-1} \mathscr{H}\left(\left|\tau_{b}\right|-C_{2}\right),
$$

where

$$
C_{1}=\frac{8}{g(s-1)}, \quad C_{2}=\Theta_{c 0} g(s-1) d .
$$

For a small bed gradient $h_{x} \ll 1$ as well as $h_{x} / \tan \phi_{s} \ll 1$, we may approximate equation (B 6) by

$$
q=C_{1} \mathscr{H}\left(\left|\tau_{b}\right|-C_{2}\right)|| \tau_{b}\left|-C_{2}\left[1+\frac{h_{x}}{\tan \phi_{s}}\right]\right|^{3 / 2} \frac{\tau_{b}}{\left|\tau_{b}\right|}\left(1-\frac{h_{x}}{\tan \phi_{s}}\right) .
$$

If the strength of the flow is far beyond critical $\left(C_{2} \ll \tau_{b}\right)$, we can approximate even further:

with

$$
q=C_{1} \sqrt{\left|\tau_{b}\right|} \tilde{\gamma}\left(\tau_{b}-\frac{3 C_{2}}{2 \tilde{\gamma} \tan \phi_{s}} h_{x}-\frac{\left|\tau_{b}\right|}{\tan \phi_{s}} h_{x}\right)
$$

$$
\tilde{\gamma}=\frac{\left(\left|\tau_{b}\right|-\frac{3}{2} C_{2}\right)}{\tau_{b}} \mathscr{H}\left(\left|\tau_{b}\right|-C_{2}\right),
$$

which can be estimated by its long-term average value. Now we can present the sediment transport as

$$
q=\alpha^{\prime} \sqrt{\left|\tau_{b}\right|}\left(\tau_{b}-\lambda_{1} h_{x}-\lambda_{2}\left|\tau_{b}\right| h_{x}\right)
$$

where

$$
\alpha^{\prime}=\frac{8 \tilde{\gamma}}{(s-1) g}, \quad \lambda_{1}=\frac{3 \Theta_{c 0} g(s-1) d}{2 \tilde{\gamma} \tan \phi_{s}}, \quad \lambda_{2}=\frac{1}{\tan \phi_{s}} .
$$

Note that (for the purposes of a linear stability analysis) both down-slope terms contribute in the same manner. The only difference is the power of the tidal shear 
stress in front of $h_{x}$. We can combine the two terms and obtain

$$
q=\alpha^{\prime} \sqrt{\left|\tau_{b}\right|}\left(\tau_{b}-\lambda^{\prime} h_{x}\right),
$$

where an estimate for the dimensional parameter $\lambda^{\prime}$ is $\lambda^{\prime} \approx \lambda_{1}+\left[\tau_{b}\right] \lambda_{2}$, and the bottom shear stress is replaced by its characteristic value, $[\tau]$. Finally, we can write down the dimensionless expressions for $\alpha$ and $\lambda$ which appear in the scaled sediment equation (2.16):

$$
\alpha=\frac{8 \tilde{\gamma}[\tau]^{3 / 2}}{(s-1) g \sigma \delta^{2}}, \quad \lambda=\frac{3 \Theta_{c 0} g(s-1) d}{2 \tilde{\gamma} \tan \phi_{s}[\tau]}+\frac{1}{\tan \phi_{s}} .
$$

Note that the first term in the expression for $\lambda$ (which is the main contribution to $\lambda$ ) makes very clear physical sense: it is equal to (grain weight/area)/flow shear stress. Typical values are $\rho=1.010^{3} \mathrm{~kg} \mathrm{~m}^{-3}, \rho_{s}=2.6510^{3} \mathrm{~kg} \mathrm{~m}^{-3}, \tan \phi_{s}=0.3$, $g=9.81 \mathrm{~m} \mathrm{~s}^{-2}$ (van Rijn 1993; Dyer 1986). Although various estimates of the critical Shields parameter on a flat bed, $\Theta_{c 0}$, are available in the literature (van Rijn 1993; Fredsoe \& Deigaard 1992), it is modelled well by a constant $\Theta_{c 0}=0.047$. The factor $\tilde{\gamma}$ is time-dependent and can roughly be approximated by its tidal average value. Only during some part of the tidal period is the shear stress larger than critical, and a rough estimate gives $0.1<\tilde{\gamma}<1$.

Now we can estimate $\alpha^{\prime}$. For typical parameters given in $\S 3$, we obtain $\alpha^{\prime} \approx$ $1.6 \times 10^{-6} \mathrm{~m}^{2} \mathrm{~s}^{-1}$. This leads to the following estimate for the characteristic time of sand wave growth: $\Delta T=1.9$ years.

The value of $\lambda$ depends on the sediment size, $d$. Typical range in which sand grain size varies is approximately from $50 \mu \mathrm{m}$ to $2 \mathrm{~mm}$, which corresponds to parameter $\lambda$ between 2.2 and 330 .

\section{REFERENCES}

Bagnold, R. A. 1956 The flow of cohesionless grains in fluids. Proc. R. Soc. Lond. A 249, 235-279. BeErens, S. B. 1995 Mixing by chaotic advection in tidal seas. PhD thesis, IMAU, Utrecht University. Bijker, R., Wilkens, J. \& Hulscher, S. J. M. H. 1998 Sandwaves: where and why. Proc. ISOPEconference Montreal.

Blondeaux, P. 1990 Sand ripples under sea waves. Part 1. Ripple formation. J. Fluid Mech. 218, $1-17$.

Dyer, K. R. 1986 Coastal and Estuarine Sediment Dynamics, pp. 180-181. Wiley.

Dyer, K. R \& HuntLey, D. A. 1999 The origin, classification and modelling of sand banks and Ridges. Cont. Shelf Res. 19, 1285-1330.

Engelund, F. 1970 Instability of erodible beds. J. Fluid Mech. 42, 225-244.

Falques, A., Calvete, D. \& Swart, H. E. DE 1998 Modelling the formation of shoreface-connected sand ridges: dynamic coupling between mean currents and topography. J. Fluid Mech. (submitted).

Fredsoe, J. \& DeigaArd, R. 1992 Mechanics of Coastal Sediment Transport, pp. 260-289. World Scientific.

Gerkema, T. 1998 A note on the effect of finite Stokes-layer thickness in a morphodynamic stability problem. In Physics of Estuaries and Coastal Seas (ed. J. Dronkers \& M. B. A. M. Scheffers), pp. 387-395. Balkema, Rotterdam.

Gerkema, T. 2000 A linear stability analysis for tidally generated sand waves. J. Fluid Mech. (in press).

Goor, S. van \& Andorka Gal, J. H. 1996 Bodem (in Dutch). Document RIKZ/OS-96.109X, 28 pp.

Hulscher, S. J. M. H. 1996 Tidal-induced large-scale regular bed form patterns in a threedimensional shallow water model. J. Geophys. Res. 101, 20727-20744.

Hulscher, S. J. M. H. \& Brink, G. M. van DEN 1999 Comparison between predicted and observed 
sand waves and sand banks in the North Sea. CTM Research Paper 99W_003/MICS_002; also submitted to J. Geophys. Res.

Hulscher, S. J. M. H., Swart, H. E. De \& Vriend, H. J. DE 1993 Generation of offshore tidal sand banks and sand waves. Cont. Shelf Res. 13, 1183-1204.

Huntley, D. A., Huthnance, J. M., Collins, M. B., Liu, C.-L., Nicholls, R. J. \& Hewitson, C. 1993 Hydrodynamics and sediment dynamics of North Sea sand waves and sand banks. Phil. Trans. R. Soc. Lond. A 343, 461-474.

Huthnance, J. 1982 On one mechanism forming linear sand banks. Estuar. Coast. Shelf Sci. 14, 79-99.

Katoh, K., Kume, H., Kuroki, K. \& Hasegawa, J. 1998 The development of sand waves and the maintenance of navigation channels in the Bisanseto Sea. Proc ICCE98, Copenhagen (to appear).

Komarova, N. L. \& Newell, A. C. 2000 Nonlinear dynamics of sand banks and sand waves. J. Fluid Mech. 415, 285-312.

LYNE, W. H. 1971 Unsteady viscous flow over a wavy wall. J. Fluid Mech. 50, 33-48.

MaAs, L. R. M. \& HAREN, J. J. M. van 1987 Observations on the vertical structure of tidal and internal currents in the central North Sea. J. Mar. Res. 45, 293-318.

MeEne, J. van DE 1994 The shoreface connected ridges along the central Dutch coast. PhD thesis, IMAU, Utrecht University; Neth. Geogr. Studies 174, KNAG, Utrecht, 256 pp.

OfF, T. 1963 Rhythmic linear sand bodies caused by tidal currents. Bull. Am. Assoc. Petrol. Geologists 47, 324-341.

Okuвo, A. \& Ozmidov, R. V. 1970 Empirical dependence of the horizontal turbulent diffusion coefficient in an ocean on the length scale. Akad. Nauk. SSSR, Izv. Atmos. Ocean Phys. 6, N.5, 534.

Ozmidov, R. V. 1968 Problems of diffusion in a sea. Akad. Nauk. SSSR, Vestnik, N.5, 220.

Pedlosky, J. 1987 Geophysical Fluid Dynamics. Springer.

PrandtL, L. 1932 Zur turbulenten Stroeming in Roehren und langs Plaetten. Ergebn. Aerodyn. Versuchsanst, Goettingen 4, 18-29 (in German).

Rijn, L. C. van 1993 Handbook of Sediment Transport by Currents and Waves. Aqua Publications, Amsterdam.

RoDI, W. 1980 Turbulence models and their application in hydraulics. IAHR Paper, $104 \mathrm{pp}$.

SoulsBy, R. L. 1990 Tidal-current boundary layers. In The Sea, Vol. 9., part A (ed. B. le Mehaute \& D. M. Hanes), pp. 523-566. John Wiley and Sons.

Swart, H. E. DE \& Zimmerman, J. T. F. 1993 Rectification of the wind-driven ocean circulation on the beta plane. Geophys. Astrophys. Fluid Dyn. 71, 17-41.

Sleath, J. F. A. 1976 On rolling-grain ripples J. Hydraul. Res. 14, 69-81.

Tennekes, H. \& Lumley, J. L. 1972 A First Course in Turbulence. MIT Press.

Visser, A. W., Sousa, A. J., Hessner, K. \& Simpson, J. H. 1994 The effect of stratification on tidal current profiles in a region of freshwater influence. Oceanol. Acta 17, 369-381.

VRIEND, H. J. DE 1990 Morphological processes in shallow tidal seas. In Residual Currents and Long Term Transport (ed. R. T. Cheng), pp. 276-301. Springer.

Wilkens, J. 1997 Sandwaves and possibly related characteristics. Report for Alkyon Hydraulic Consultancy and Research. 